\title{
Microscopic and Microstructural Study of Arsenic Copper from Spidezh of Bazman
}

\author{
Vahid Pourzarghan $^{1 \star}$, Hamidreza Bakhshandefard ${ }^{2}$, Mohammad Heydari ${ }^{3}$ \\ ${ }^{1}$ PhD Candidate in Conservation \& Restoration of Historical Artifacts, Assistant Professor, Faculty of \\ Applied Arts, Zabol, IRAN \\ ${ }^{2}$ Assistant Professor, Department of Conservation of Cultural and Historical Artifacts, Art University of \\ Isfahan, IRAN \\ ${ }^{3}$ Administration of Cultural Heritage of Sistan \& Balochestan, Zahedan, IRAN
}

\begin{abstract}
Undoubtedly, one of the most important man-made objects in history is copper objects and their alloys, and in this regard, according to the available evidence, its oldest alloy, as copper-arsenic, has an important role in history. The existence of these works in various sites, especially in southeastern Iran since the Early Bronze Age, confirms this claim. One of the most significant strategic sites is located in the southeastern of Iran, is Spidezh. It is one of the most important settlement centers belonging to the third and second millennium BC, which is located $40 \mathrm{~km}$ southwest of Bazman. The site is located $3 \mathrm{~km}$ from Makhsan village and about $160 \mathrm{~km}$ from Iranshahr. Significant artifacts were brought to light from Spidezh cemetery such as stamps, ornaments (beads, pins, kohl holder pin, mirrors), agricultural tools, different types of weapons, etc. Spidezh registered under the number 6745 in the list of national monuments of Iran. In this study, a number of metal objects in Spidezh cemetery were examined to identify copper-arsenic compounds and the properties of this alloy. To study these objects, the SEM-EDS method was used to identify the microstructure of the metal matrix and the chemical composition of the elements, as well as the metallographic method to study the fabrication technique and phases studies. SEM-EDX analysis shows that some objects are made of $\mathrm{Cu}-\mathrm{As}-\mathrm{Zn}$ alloy and others are made of $\mathrm{Cu}$-As alloy by casting and are considered as arsenic-containing bronze alloys. Arsenic acts as an excellent oxidant element in metal, reducing the porosity of the alloy and significantly increasing its hardness. Deliberate addition of low levels of arsenic can act as a dioxin in the samples. Also, the presence of sulfide in the impurities of these objects can be due to the use of sulfide ores. The amount of calcium and potassium in the EDS spot analysis of the inclusions indicates that this amount of elements is owing to coal fuel. These elements are considered as flux charge melt and eventually absorb the slag. The SEM images suggest that the impurities in the whole matrix of the metal in a point and elongated from can be the reason for the presence of unrefined copper with spiess in the process of making these works. This discarded waste contains sulfide, arsenide and iron-like compounds. The uniform and high percentage of 5\% arsenic in these works shows that the manufacturing process of these alloys has been done by Co-Smelting method. Metallographic studies show that the physical operation on these works was a repetition of a cycle of cold work and annealing (irradiation) to further shape these works.
\end{abstract}

Keywords: Ancient metalworking, Arsenic copper, Co-smelting, Chance metallurgy, Spidezh of Bazman, $\mathrm{Cu}-\mathrm{As}$

\footnotetext{
* Corresponding author: vahidpourzarghan@gmail.com

Copyright $(2020$, the Authors / This open-access article is published under the terms of the Creative Commons Attribution-NonCommercial 4.0 International License which permits Share (copy and redistribute the material in any medium or format) and Adapt (remix, transform, and build upon the material) under the Attribution NonCommercial .terms
} 


\section{Introduction}

Copper alloy containing arsenic is a product of man-made production in the early $5^{\text {th }}$ millennium BC [1]. There are many sites in the Iranian plateau from the Early Bronze Age that have been studied. areas are Tappeh Yahya, Shahdad and Meymantabad (25 km southeast of Tehran) could be consider as typical site in this regard. The alloys obtained from these site have been analyzed using ICP-MS and PIXE to show that they are arsenical alloy [2,6]. One of the most important and strategic sites on the Iranian plateau, which is located in the southeastern region of Iranshahr (Fig. 1, A-B), is the Spidezh of Bazman, dating back to third and second millennium BC [12]. The main purpose of this study is to investigate the microstructure and fabrication of metal artifacts discovered from Spidezh.

\section{Materials and Methods}

The objects studied in this come from Spidezh of Bazman cemetery excavation during 2002-2006 seasons which contains objects such as two axes (1-M, 2-A) and one dagger (3-D). A small crosssectional sample of each object was prepared for instrumental analysis and metallography. The samples were then mounted in epoxy resin. To prepare the samples, they were sanded and polished using 800 to 4000 -degree sandpaper. Finally, diamond $(1 \mu \mathrm{m})$ paste was used for the final polishing. The samples were etched in $\mathrm{FeCl}_{3}$ alcoholic solution and examined under microscope (OM). In order to identify the chemical composition of studied samples, mounted specimens were made by SEM-EDX method in Razi Metallurgy Laboratory of Tehran. This analysis was performed with a scanning electron microscope (SEM) model VEGA II, made by TESCAN Company in the Czech Republic and an X-ray spectrophotometer (EDS) device made in RONTEC, Germany with Quntax software model QX $\mathrm{Q}_{2}$

\section{Result and Discussion}

The results of SEM-EDS analysis on dagger (3-D) and two other analyses (2-A, 1-M) from Spidezh are shown in (table-1). These articles are made with copper base and with a high percentage of $5 \%$ arsenic by Co-Smelting method. The element copper in these works varies from 88.5 to $72.75 \%$ and arsenic from 1.6 to $4.6 \%$ in sample matrix. The $2 \%$ arsenic content in the samples can cause discoloration of the surface, creating a silver surface [2]. Low level of arsenic can play a role in dioxide of these artifacts. The element zinc in two axes varies from 1.25 to $3.20 \%$. This percentage of zinc can be due to presence of zinc-containing ores such as smithsonite $\left(\mathrm{ZnCO}_{3}\right)$, sphalerite $(\mathrm{ZnS})$ and rosasite $(\mathrm{Cu}, \mathrm{Zn}) 2 \mathrm{CO} 3(\mathrm{OH}) 2$ [14] in the observed objects. The main composition of these two axes is $\mathrm{Cu}-\mathrm{As}-\mathrm{Zn}$. Also, the high percentage of 2 to $7.5 \%$ of iron in the samples of axes (table-1) is most likely due to the presence of completely refined copper. Identifying this percentage of iron in a point can be the reason for using spiess to produce spiess from smelting iron ore such as arsenopyrite $(\mathrm{FeAsS})$, lölligite $\left(\mathrm{FeAsS}_{2}\right)$ and scorodite $\left(\mathrm{FeAsO}_{4} \cdot 2 \mathrm{H}_{2} \mathrm{O}\right)[8,20,21,22]$. In the image of SEM from the Axe (1-M) in image 4 (B), the stretched veins of iron impurities along the object are the result of hammering in this work, it is possible that these iron impurity particles could be crushed or widened during the hammering, which is quite evident in figure 4, 5(B). The dagger (3D) has no sound metal. Metallographic images of axes after etching with $\mathrm{FeCl}_{3}$ alcohol solution, microstructure are observed in a complex and scaly from with many strain lines, which is due to the hard work on these works. Metallographic examination of figure -8 (A, B) shows that the samples were formed during a cycle with hot and cold work operations and the slip lines in these samples show that the final operation was done with cold work. The twin lines are also smooth and elongated and scattered in the metallographic images $8(\mathrm{~A}, \mathrm{~B})$ due to the hard work on these samples. According to figure -1 (B) the phase diagram under casting conditions. One phase may occur during arsenic solidification, which is phase $\alpha-(\mathrm{Cu}-\mathrm{As})$, which is present in the two axes.

\section{Conclusion}

According to the SEM-EDS analysis carried out on the objects, it was determined that the axes discovered from Spidezh were made of alloy $\mathrm{Cu}-\mathrm{As}-\mathrm{Zn}$ and the dagger objects were made of alloy $\mathrm{Cu}$-As by casting in the mold. Due to the high volatility of arsenic, the concentration of this material in casting has not been easily controlled and has reduced arsenic. Also in higher formability stage with heat treatment (Annealing) has caused more arsenic drop from solid solution. Another 
feature of arsenic is that it has been used as an alloy component with copper to produce copper containing arsenic. 


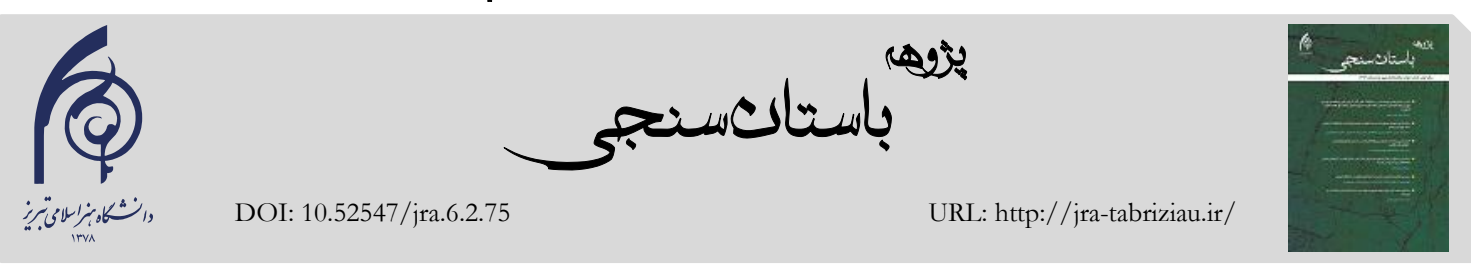

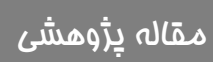

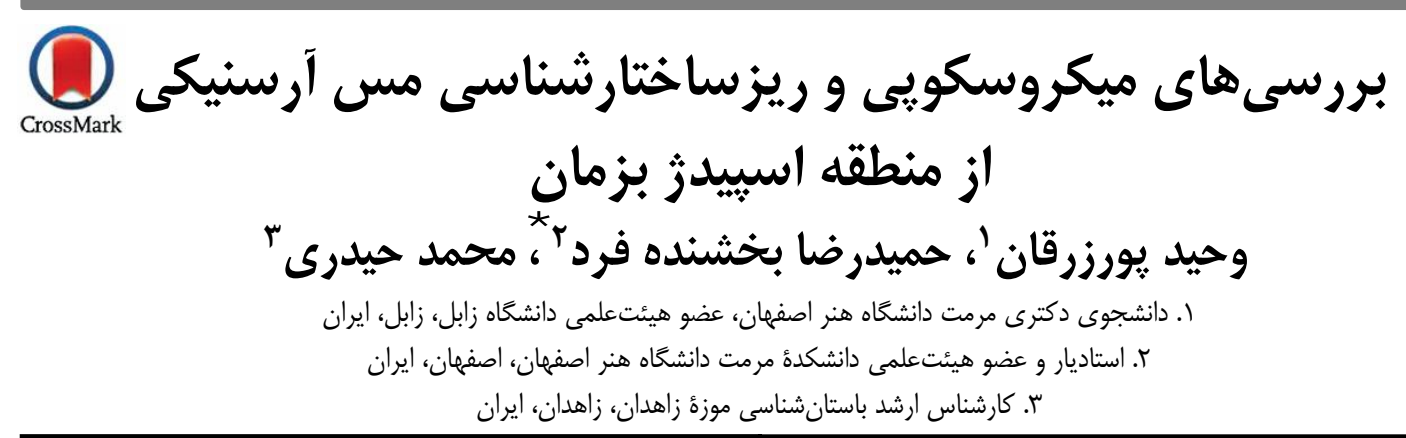

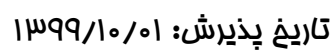

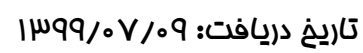

جكيده

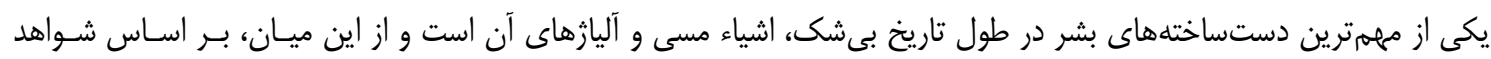

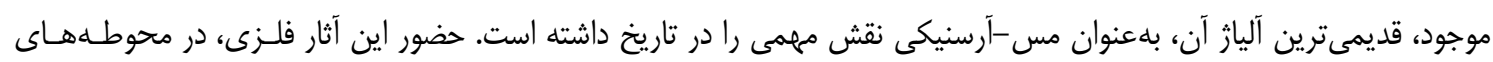

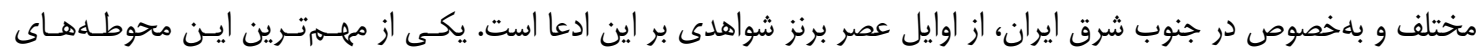

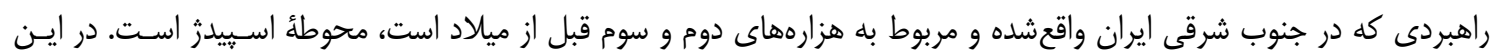

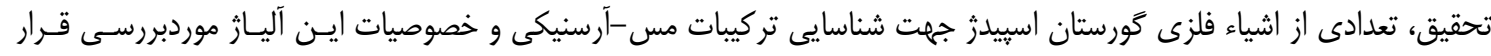

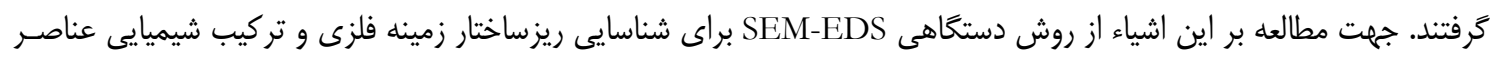

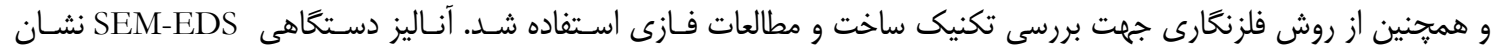

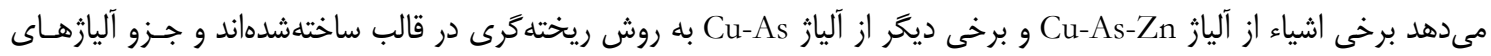

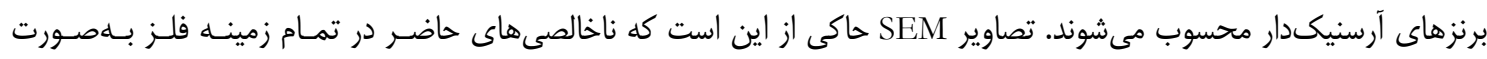

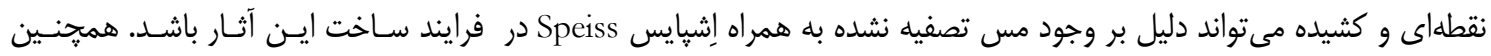

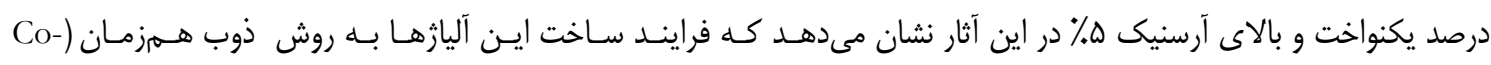

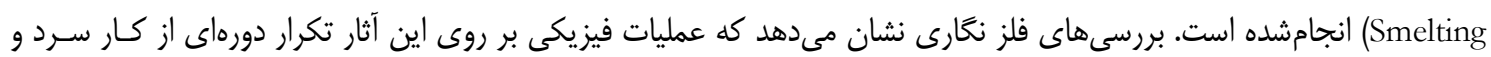

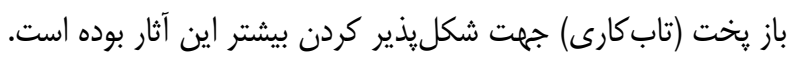

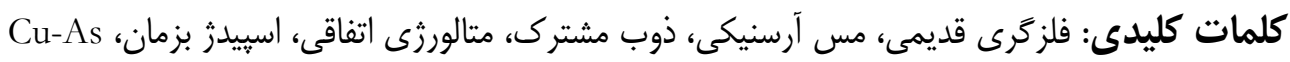

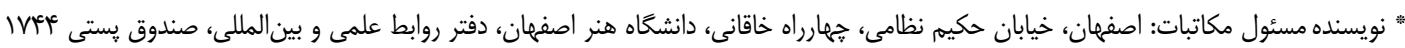
يست الكترونيكى: HR.Bakhshan@aui.ac.ir

C) حق نشر متعلق به نويسنده(كان) است و نويسنده تحت مجوز Creative Commons Attribution License به مجله اجازه مىدهد مقاله خاب شده را با ديكران به اشتراك بكذارد منوط بر اينكه حقوق مؤلف اثر حفظ و به به انتشار اوليه مقاله در اين مجله اشاره شود. 
سنگ معدن مىشد [2].

د) ذوب همزمان كانههـاى سـولفيدى و اكسـيدى از معاز

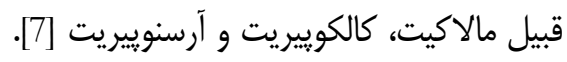

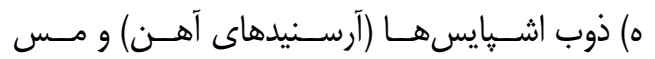

از مهمترين مناطق عصر برنز در فلات ايران، منطقة

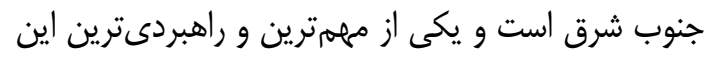

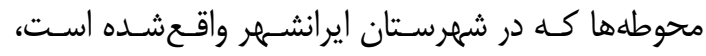
محوطة باستانى اسيبيدز بزمان متعلق به هزارة سـوم و دوم ق.م است. در اين محوطه كه توسط محمد حيدرى مـورد

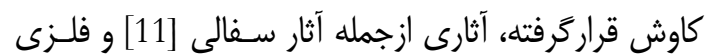

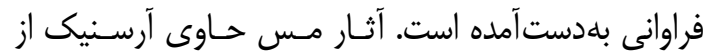

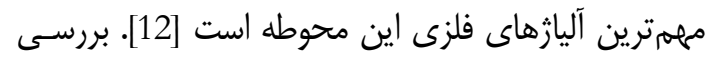

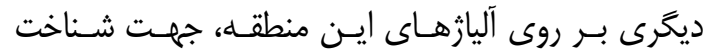
تركيب شيميايى و روش ساخت آنها انجامنشده است. بـاــاـ

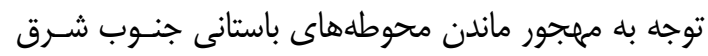
ايران و در دسترس نبودن اطلاعات كافى از متـالورزى و جنابـ

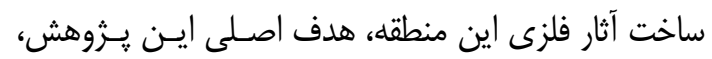
بررسى ريزساختار و ساخت آثار فلزى مكشـوفه از اسـيبدز بزمان است.

\section{معرفى منطقة اسبيدز بزمان}

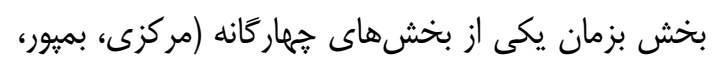

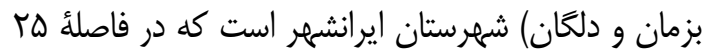

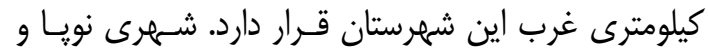

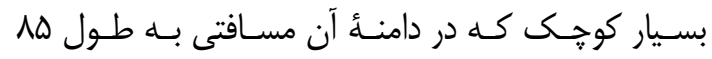

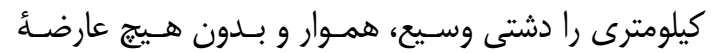

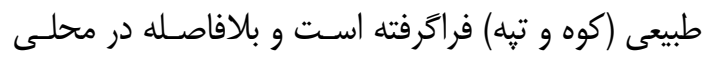

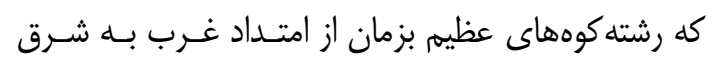

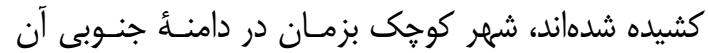

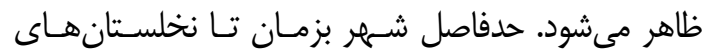
اسبيدز حدود سأ كيلومتر است [11].

\section{محوطة اسيبدز}

اسبيدز يا اسيبدز (دز سفيد)، نام خود را از قلعهاى بـ به همين

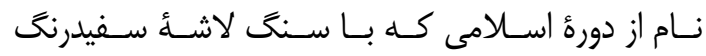

\section{(. ) مقدمه}

آلياز مس حاوى آرسنيك، نخستين محصول سـاختهشـدة

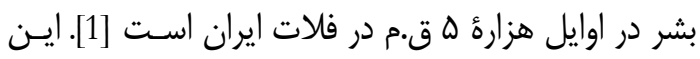
آلياز در هزارهٔ جهارم و اوايل هزارة سوم ق.م نيز در مركـز

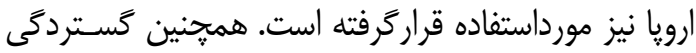

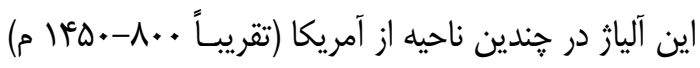

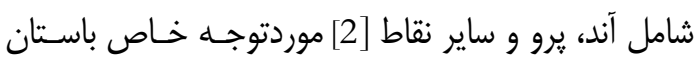
شناسان و تاريخدانان قرارگَرفته است. در فلات ايران سايتهاى زيادى از اوايل عصر برنز Early Bronze Age قراركرفتهاند. يكى از مهمترين اين محوطهها، محوطهُ تِّه

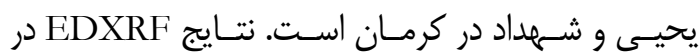
بررسى اوليه و مطالعات آركئومتالورزى بر يافتههـاى ايـن

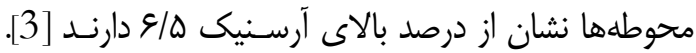

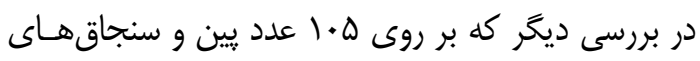

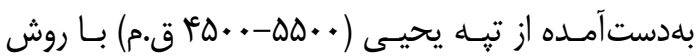

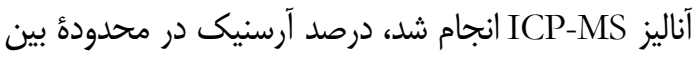

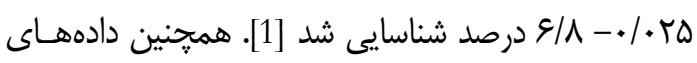

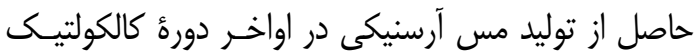

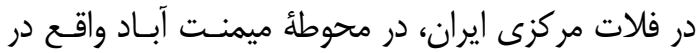

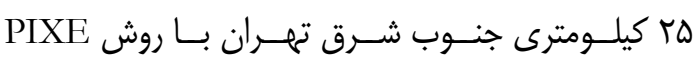

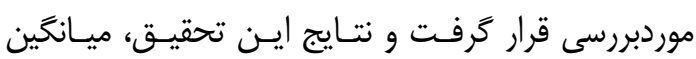

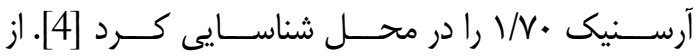

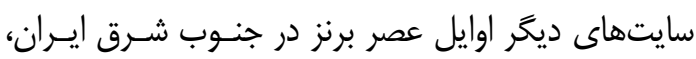
محوطة شهر سوخته است. نتايج مطالعـات متـالورزى بـر بـر

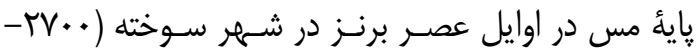

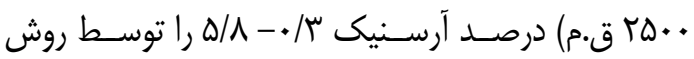

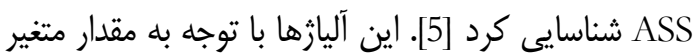

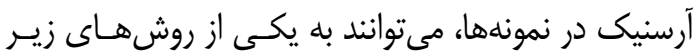

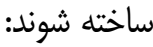
الف) تركيب مس طبيعى با مواد معدنى آرسنيد-مس

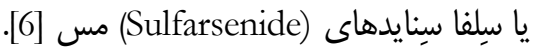
ب) ذوب كانههاى مس غنى از آرسنيك

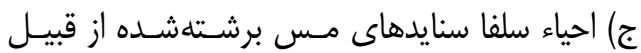

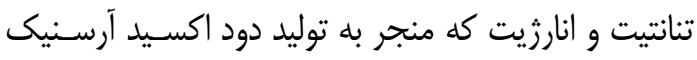
سمى و از دست دادن بخش زيادى از آرسنيك موجـود در 

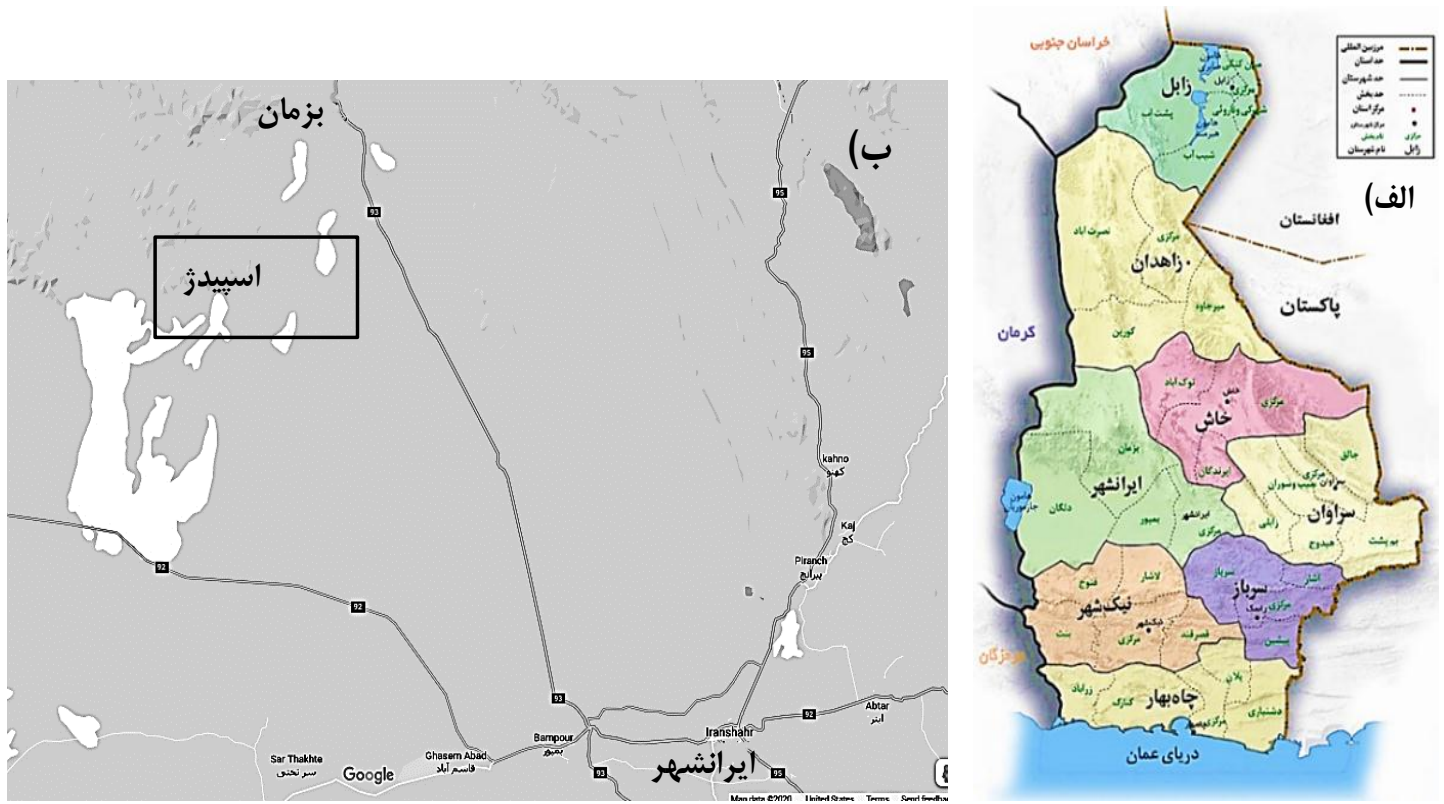

شكل ا: الف: موقعيت شهرستان ايرانشهر در جنوب شهرستان سيستان و بلوجستان ب: ماهوارهاى از موقعيت گورستان اسيبدز مربوط به هزارهٔ دوم و سوم ق.م

Fig. 1: A: Location of Iranshahr in the south of Sistan and Baluchestan. B: Satellite image of the Spidej cemetery related to the second and third millennium BC

و هزارهٔ دوم قبل از ميلاد، سطح دانش و فنّاورى بـالايى

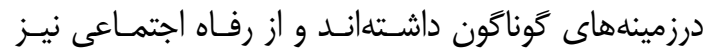
بمرهمند بودهاند [11].

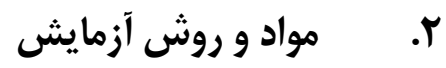 معرفى نمونههاى مورديزوهش}

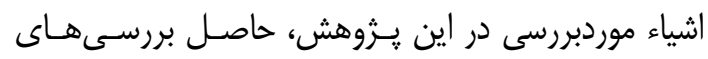

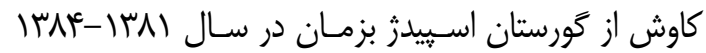

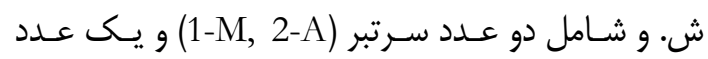
خنجر (3-D) است.

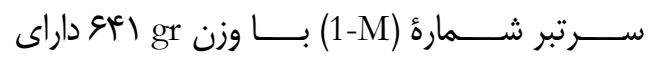

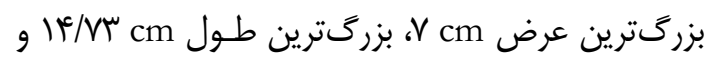

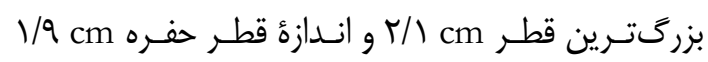

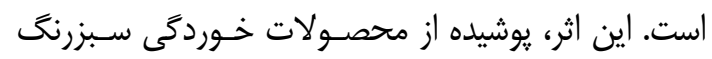

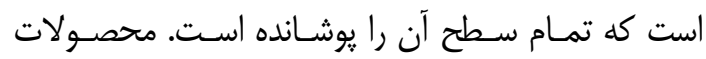

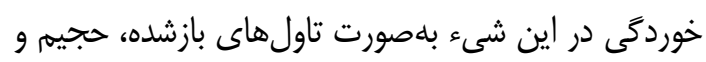

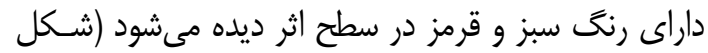
.(A, B, C r وجود لايههاى كِل نيز در بعضى از نقاط اين اثر ديده

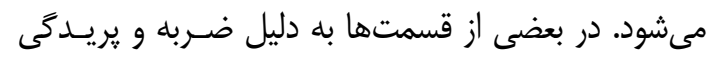
VV
ساختهشده، كرفته و يكى از مهمترين كـانونهـا و مراكـز

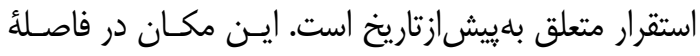

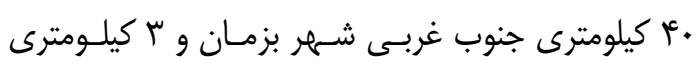

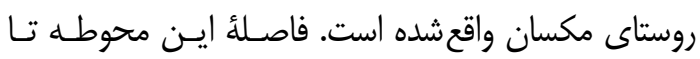

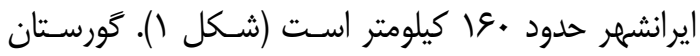

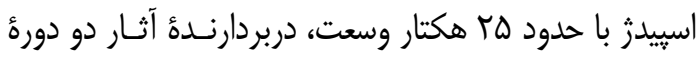

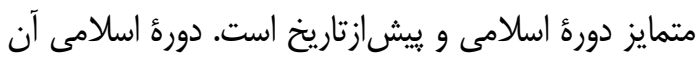

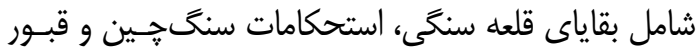
است كه اغلب در جنوب شرق محوطه واقعشده است. آثار

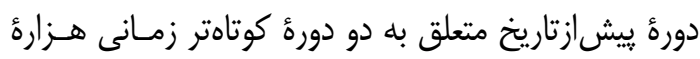

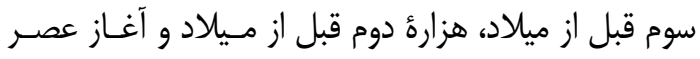

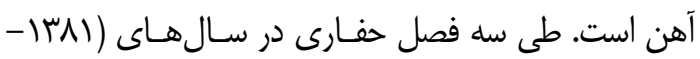

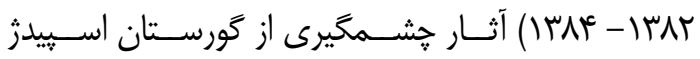

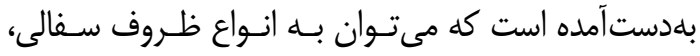

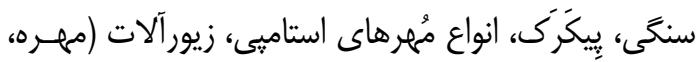

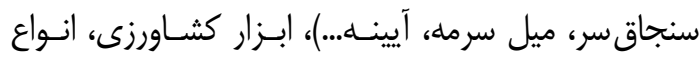

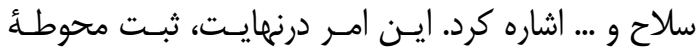

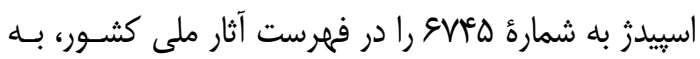

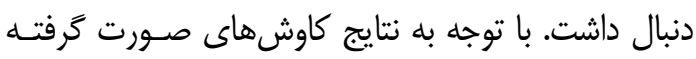

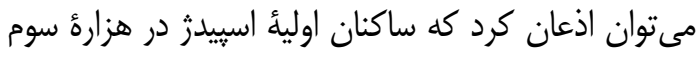


يوشــيده شـــه اسـت. در قسـمت هلالسى شـكل تبـر،

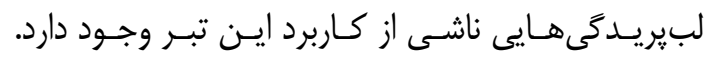

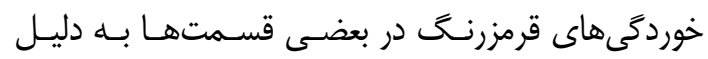

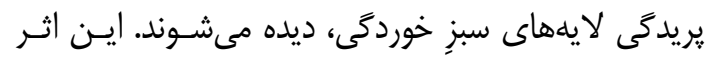

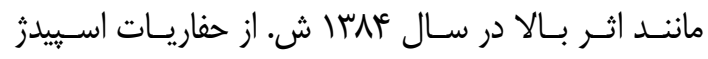

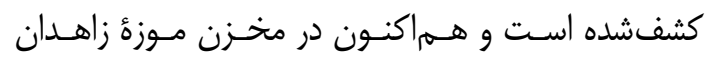

$$
\text { نخمهدارى مىشود. }
$$

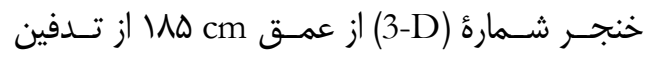

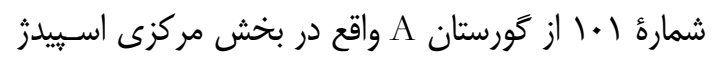

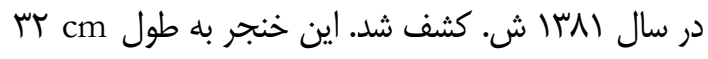

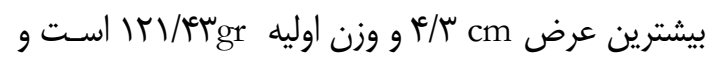

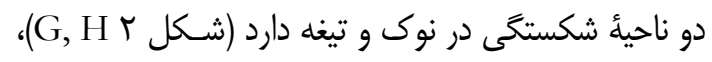

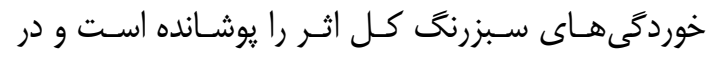
بعضى از قسمتها، كل ولاى محيط دفن بر اثر باقيمانـده

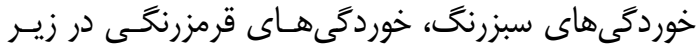

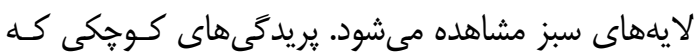

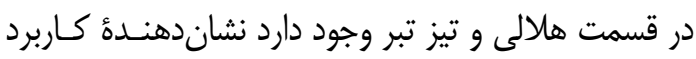

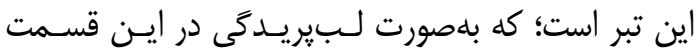

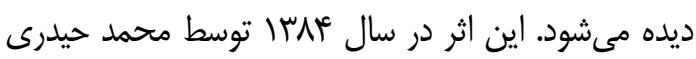

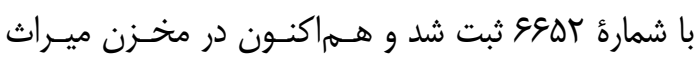

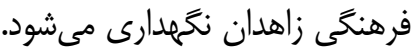

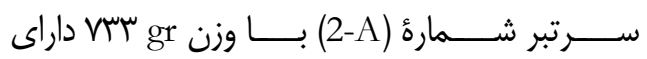

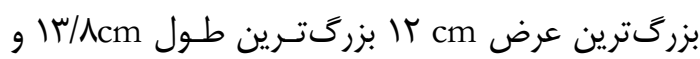

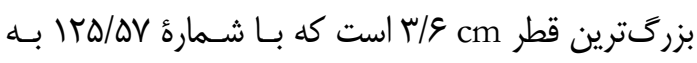

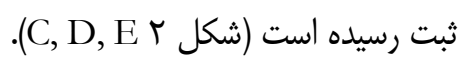

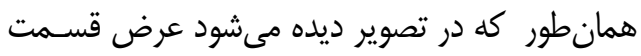

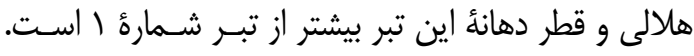

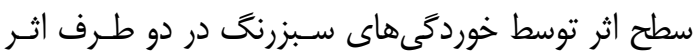
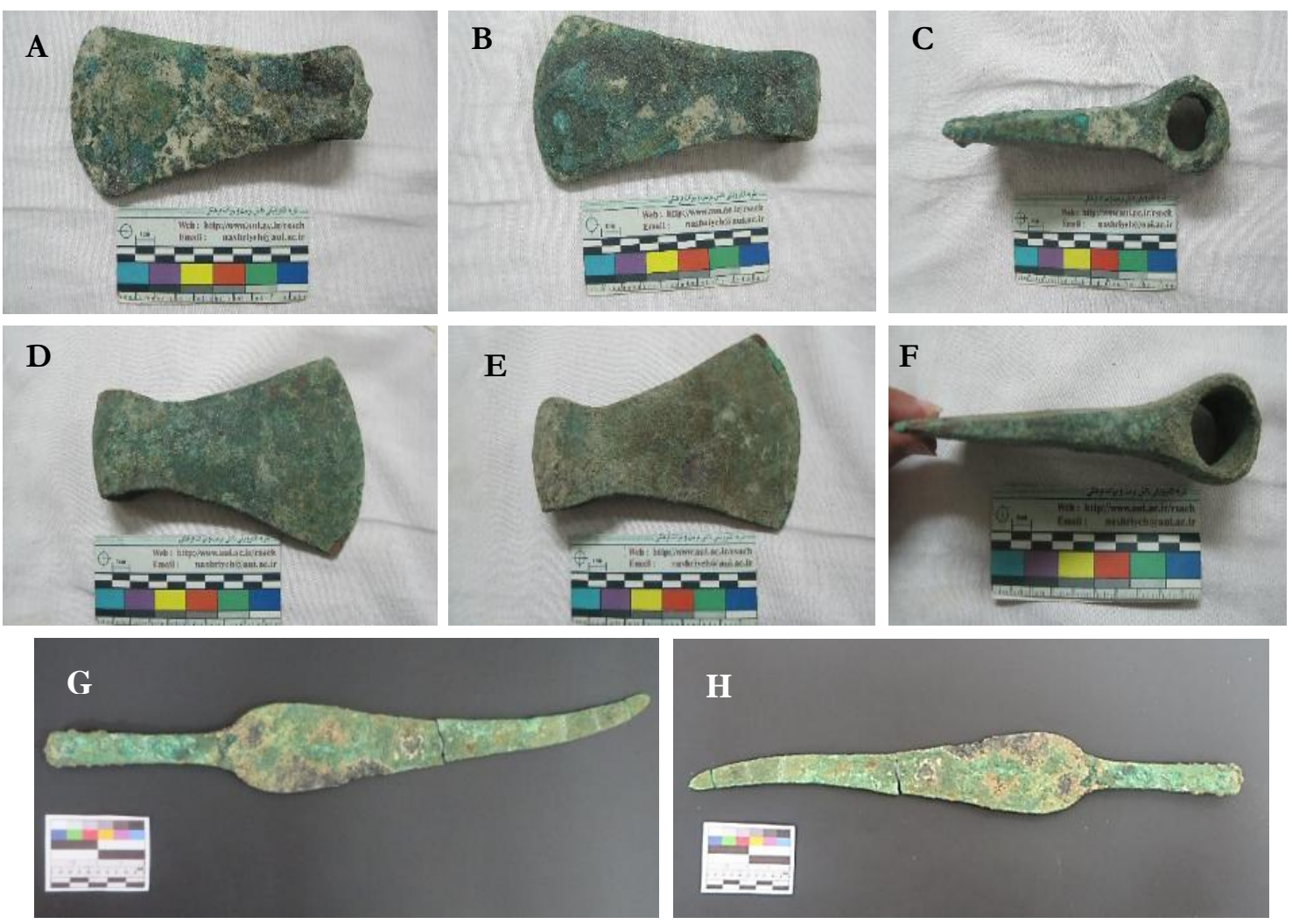

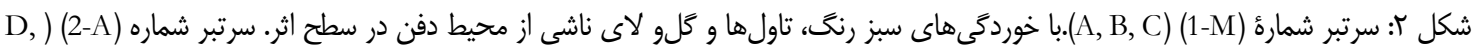

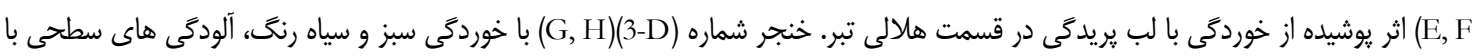

$$
\text { تيغه شكسته شده }
$$

Fig. 2: Axe head (1-M) (A, B, C). with green corrosion, blisters and mud from the burial environment on the work surface. Axe head No (2-A) (D, E, F) effect covered with corrosion which chipped in the crescent part of the axe. Dagger No (3-D) $(\mathrm{G}-\mathrm{H})$ with green and black corrosion, surface contamination with broken blade 
است. در قسمت دسته، آسيبهايى بلهـورت تـركىهـاى انجام شد.

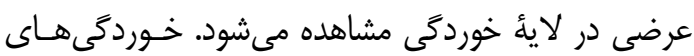

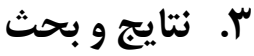
روش SEM-EDS بهكار رفنه، يك آناليز نيمه كمّى اسـت

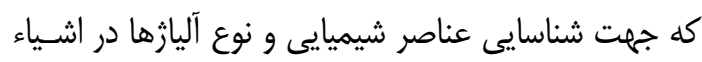

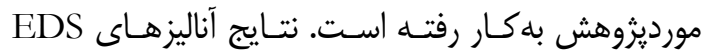
(2-A بر روى يك خنجر (3-D) و دو تبر (-D) SEM-

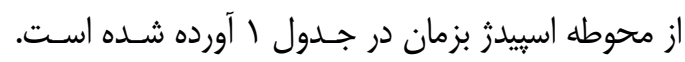

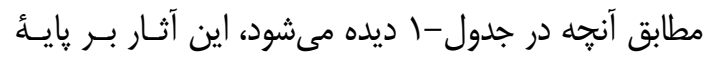

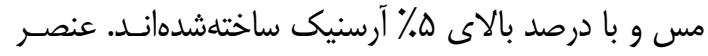

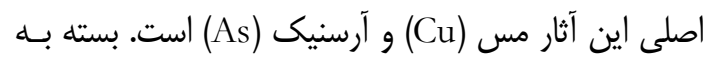

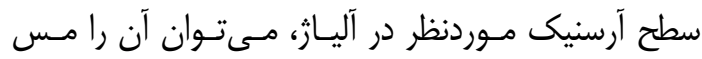

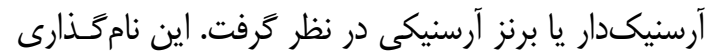
بر اساس مقدار آرسنيك در مس انجام مىشودي؛ كه لـتمن،

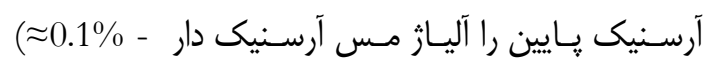
20.5\% As) (2) - 7\% As)

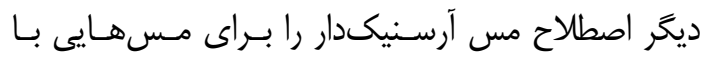

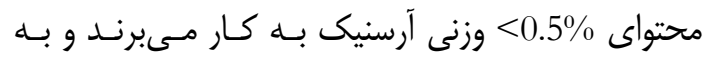
مسهايى با 0.5 ج وزنى آرسنيك، برنز آرسنيكدار كَفتـه

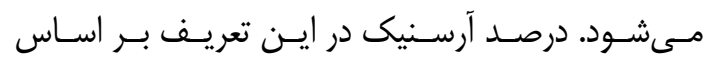
رسانندگى مس تعريفشده است [10].

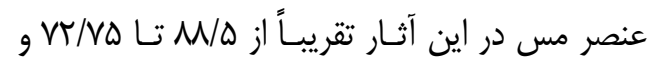

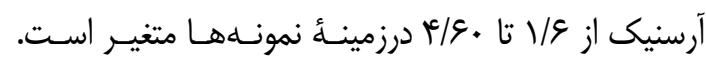

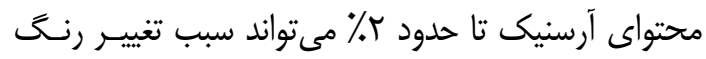

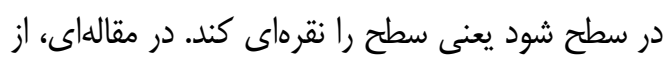

سياه و زغالى رنغ در دو طرف تيغه ديده مى شوند. اين اثر مثرد

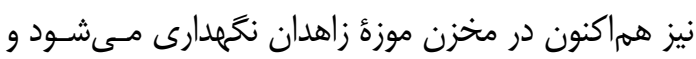

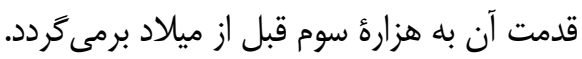

\section{روش آمادهسازى نمونهها}

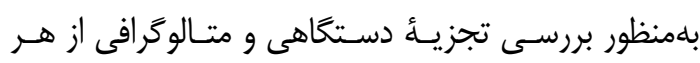

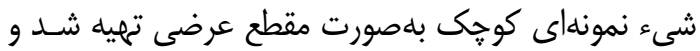

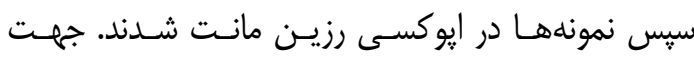
آمادهسازى، نمونهها با استفاده از سنبادههايى با درجهُ ...

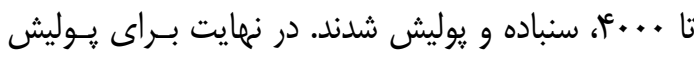

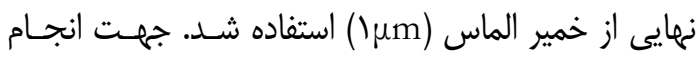

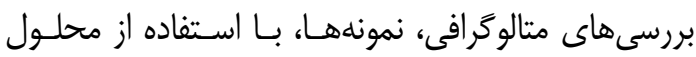
الكلى

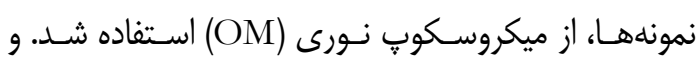
سبس بوسيله ميكرسكوٍ نورى (OM) مورد بررسى قرار كرفتند.

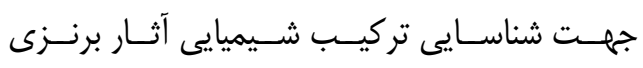
مورديزوهش از نمونههاى مانت شده و با روش دستخاهى ترى SEM-EDS

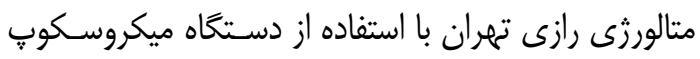

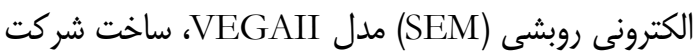

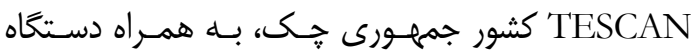

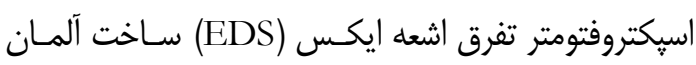
مدل RONTEC، با نرمافزار QUNTAX مـدل Qـل

جدول ا: نتايج آناليز SEM-EDX از سه شىء برنزى مس آرسنيكى بهدست آمده از كورستان اسِييدز (wt\%)

Table 1: Results of analysis SEM-EDX of three Copper arsenic-containing artifacts obtained from Spidej cemetery

\begin{tabular}{|c|c|c|c|c|c|c|c|c|c|c|c|c|c|}
\hline Sample & $\mathbf{O}$ & $\mathrm{C}$ & $\mathrm{Cl}$ & $\mathbf{K}$ & $\mathrm{Ca}$ & $\mathrm{Fe}$ & $\mathrm{Cu}$ & $\mathrm{Zn}$ & As & Sb & $\mathrm{Si}$ & $\mathrm{S}$ & Ag \\
\hline 1-M-Mon $\mathbf{A}$ & 20.36 & - & 8.05 & 2.45 & 0.51 & 4.99 & 33.48 & 3.22 & 3.57 & - & - & - & - \\
\hline 1-M $\mathrm{M}_{1}$-Zon $\mathbf{B}$ & 26.79 & - & 3.31 & 2.27 & - & 7.36 & 20.57 & - & 0.07 & - & 0.99 & 2.89 & - \\
\hline 1-M $\mathrm{M}_{1}$-Matrix & - & - & - & - & - & - & 88.49 & 3.20 & 4.10 & - & - & - & - \\
\hline $2-A_{2}-Z o n \mathbf{A}$ & 16.77 & - & 10.65 & - & 0.18 & 1.72 & 33.28 & - & 0.07 & 2.65 & - & 4.14 & - \\
\hline 2-A2-Zon $\mathbf{B}$ & 12.18 & - & 9.93 & 0.31 & - & 1.97 & 30.26 & - & 0.02 & 2.81 & - & 4.42 & - \\
\hline 2- $\mathrm{A}_{2}$-Matrix & - & - & - & - & - & - & 82.13 & 1.25 & 4.60 & - & - & - & 0.24 \\
\hline 3-D-Matrix & 12.55 & 2.63 & 8.72 & - & - & - & 72.75 & - & 1.59 & - & - & - & - \\
\hline 3-D-Zone A & 10.34 & - & 5.11 & - & - & - & 82.56 & 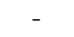 & - & - & - & - & - \\
\hline 3-D-Zone B & 15.62 & 1.90 & 15.78 & - & 0.92 & - & 62.37 & - & 5.38 & - & - & - & - \\
\hline 3-D-Zone C & 14.11 & 13.51 & 7.97 & - & - & - & 41.15 & - & 2.79 & - & 1.27 & - & - \\
\hline
\end{tabular}

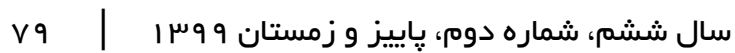


فرايند ذوب دارد. اين عنصر در اوايـل عصـر مـس تقريبـاً

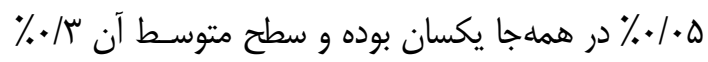

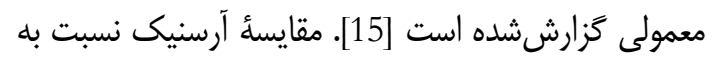

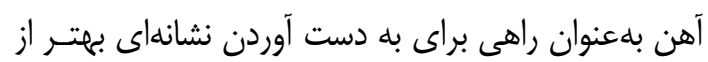

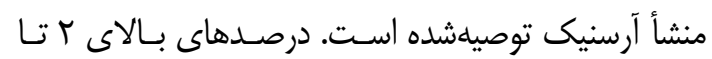

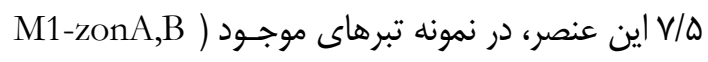

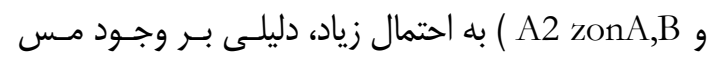

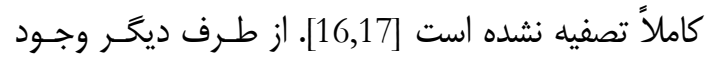
ناخالصىهاى آهن بلهصورت يراكنده در نمونه تبـر شـماره (A2 zonA,B و M1-zonA,B)

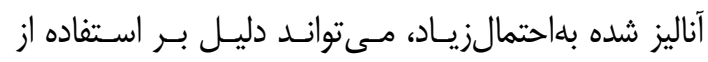

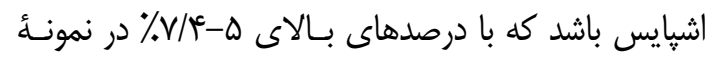
A2 ZonA,B و و M1-zonA,B شناسايى شده است. اشيايس در حقيقت تركيبات سولفيدى

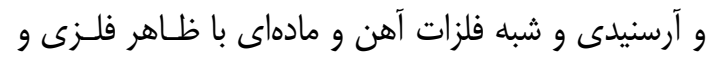

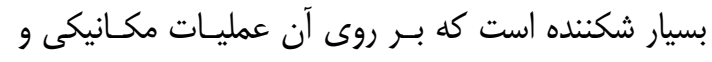

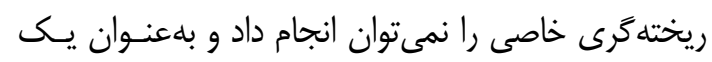

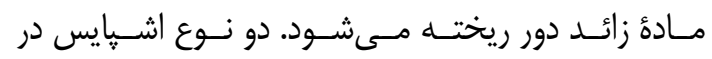

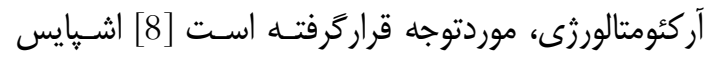

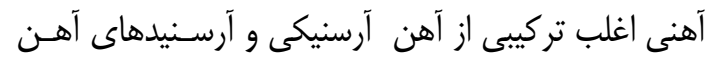

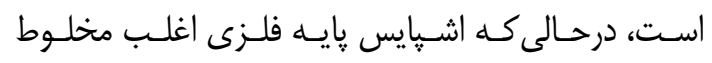

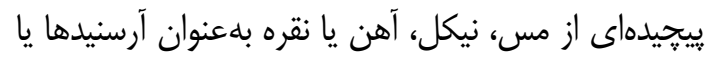

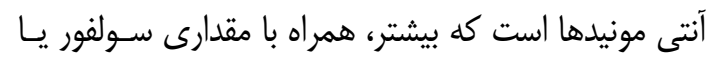

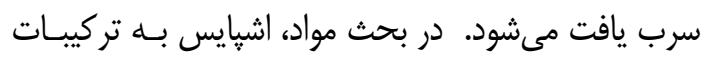

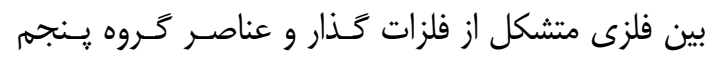

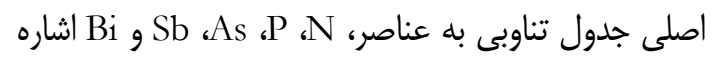

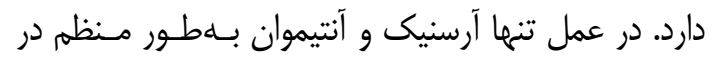

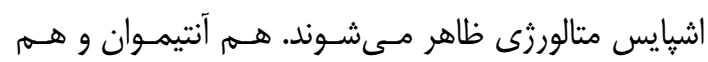

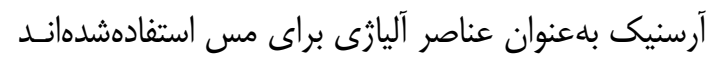

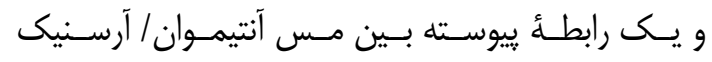
(آرسنيك و آنتيموان در محلول جامد در فاز مس بـ آلفا قـرار

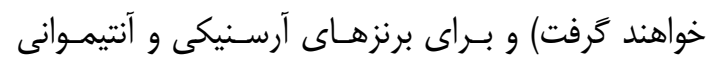

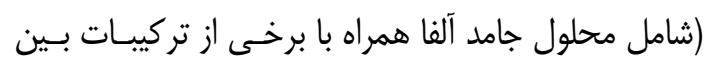

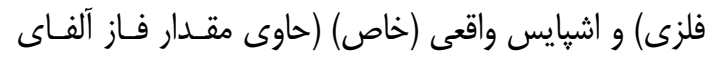

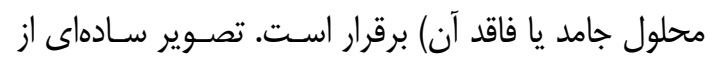

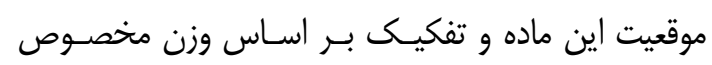

اين بِديده با لفظ مس كثيف ياد شده اسـت كـه از همـين

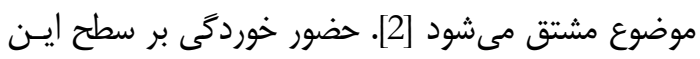

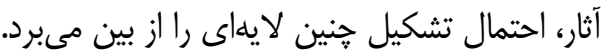

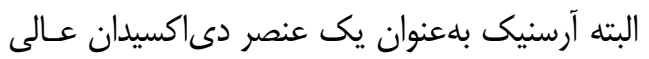
در فلز عمل مى كند كه سبب كاهش تخلخل آلياز مىشود

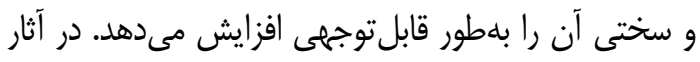

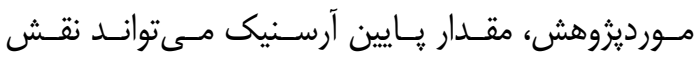
دى اكسيدان در نمونهها را داشته باشد [13].

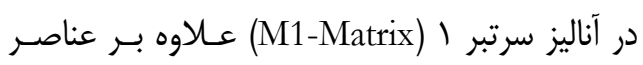

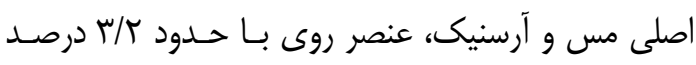

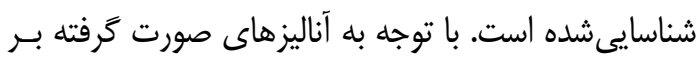

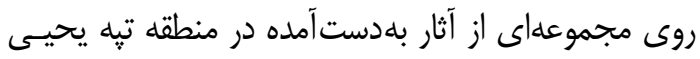

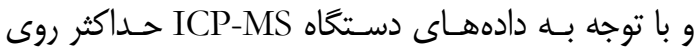

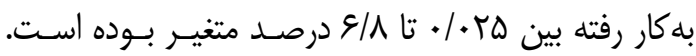

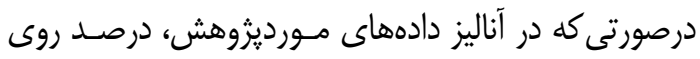

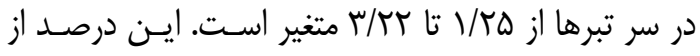

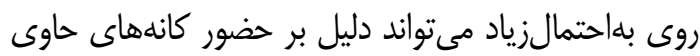
Sو روى مانتــ (Smithsonite $\left(\mathrm{ZnCO}_{3}\right)$ در (Rosasite (Cu, $\mathrm{Zn})_{2} \mathrm{CO}_{3}(\mathrm{OH})_{2}$ [14] يـ (ZnS) شىء مورد نظر باشد. استفاده از آلياز بـرنج در تيـه يحيسى

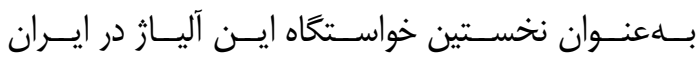

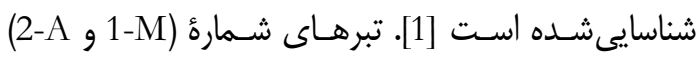

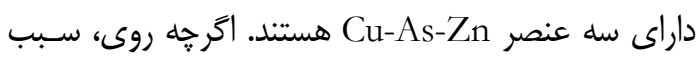

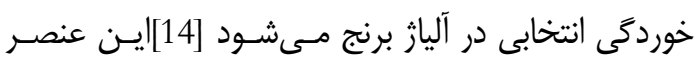

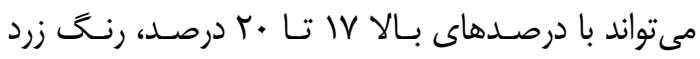

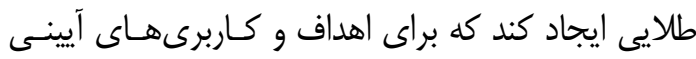

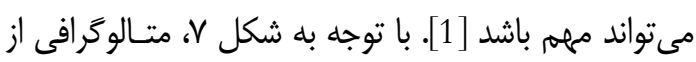
نمونه، نشان از مغز فلزى سالم در اين دو اثر دارد. اكسيثن

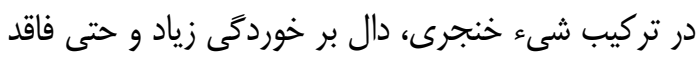

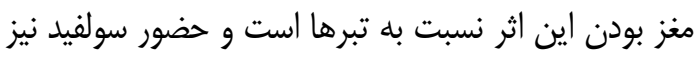

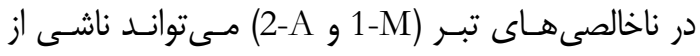

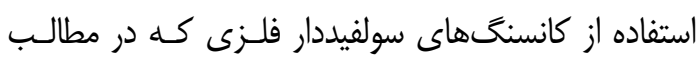

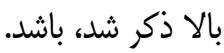
در بـين تمـامى عناصـر، عنصـر جزئسى و كسم كـهـ

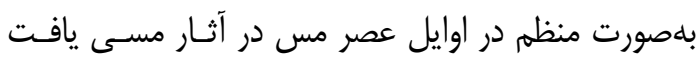

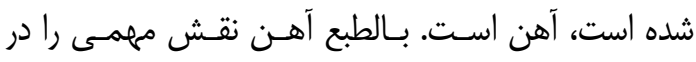


جدول ז: رايجترين مواد معدنى حاوى آرسنيك [23].

Table 2: The most common minerals containing arsenic

\begin{tabular}{|c|c|c|}
\hline 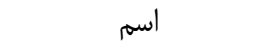 & ماده معدنى & فرمول \\
\hline \multirow{9}{*}{ Sulfides and solfosalts } & Arsenopyrite & FeAsS \\
\hline & Arsenical pyrite & $\mathrm{Fe}(\mathrm{As}, \mathrm{S})_{2}$ \\
\hline & Cobaltite & CoAsS \\
\hline & Enargite & $\mathrm{Cu}_{3} \mathrm{AsS}_{4}$ \\
\hline & Gersdorffite & $\mathrm{NiAsS}$ \\
\hline & Orpiment & $\mathrm{As}_{2} \mathrm{~S}_{3}$ \\
\hline & Proustite & $\mathrm{Ag}_{3} \mathrm{AsS}_{3}$ \\
\hline & Realger & $\mathrm{As}_{4} \mathrm{~S}_{4}$ \\
\hline & Tennantite & $(\mathrm{Cu}, \mathrm{Fe})_{12} \mathrm{As}_{4} \mathrm{~S}_{13}$ \\
\hline \multirow[t]{6}{*}{ Arsenides } & Domeykite & $\mathrm{Cu}_{3} \mathrm{As}$ \\
\hline & Löllingite & $\mathrm{FeAs}_{2}$ \\
\hline & Nickeline & NiAs \\
\hline & Rammelsbergite & $\mathrm{NiAs}_{2}$ \\
\hline & Safflorite & $\mathrm{CoAs}_{2}$ \\
\hline & Sperrylite & $\mathrm{PtAs}_{2}$ \\
\hline \multirow[t]{6}{*}{ As (III) Oxides } & Arsenolite & $\mathrm{As}_{2} \mathrm{O}_{3}$ \\
\hline & Claudite & $\mathrm{As}_{2} \mathrm{O}_{3}$ \\
\hline & Gebhardite & $\mathrm{Pb}_{8}\left(\mathrm{As}_{2} \mathrm{O}_{5}\right) \mathrm{OCl}_{6}$ \\
\hline & Leiteite & $\mathrm{ZnAs}_{2} \mathrm{O}_{4}$ \\
\hline & Reinerite & $\mathrm{Zn}_{3}\left(\mathrm{AsO}_{3}\right)_{2}$ \\
\hline & Trippkeite & $\mathrm{CuAs}_{2} \mathrm{O}_{4}$ \\
\hline \multirow[t]{9}{*}{ As $(V)$ oxides } & Austinite & $\mathrm{CaZnAsO}_{4} \mathrm{OH}$ \\
\hline & Conichalcite & $\mathrm{CaCuAsO}{ }_{4} \mathrm{OH}$ \\
\hline & Erythrite & $\mathrm{Co}_{3}\left(\mathrm{AsO}_{4}\right)_{2} .8 \mathrm{H}_{2} \mathrm{O}$ \\
\hline & Hörensite & $\mathrm{Mg}_{3}\left(\mathrm{AsO}_{4}\right)_{2} .8 \mathrm{H}_{2} \mathrm{O}$ \\
\hline & Juhenbaumite & $\mathrm{Ca}_{5}\left(\mathrm{AsO}_{4}\right)_{3} \mathrm{OH}$ \\
\hline & Mansfieldite & $\mathrm{AlAsO}_{4} \cdot 2 \mathrm{H}_{2} \mathrm{O}$ \\
\hline & Oliverite & $\mathrm{Cu}_{2}\left(\mathrm{AsO}_{4}\right) \mathrm{OH}$ \\
\hline & Sarmientite & $\mathrm{Fe}_{2} \mathrm{AsO}_{4} \mathrm{SO}_{4} \mathrm{OH} .5 \mathrm{H}_{2} \mathrm{O}$ \\
\hline & Scorodite & $\mathrm{FeAsO}_{4} \cdot 2 \mathrm{H}_{2} \mathrm{O}$ \\
\hline
\end{tabular}

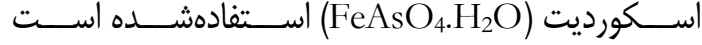
[8,20,21,2,22]. در جدول شماره ا فهرسـت رايـجتـرين مواد معدنى حاوى آرسنيك آورده شده است.

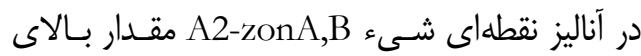

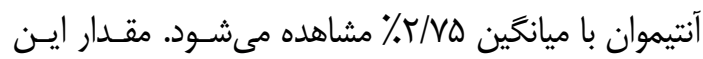

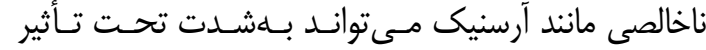

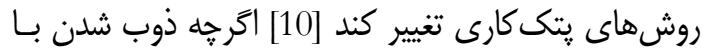

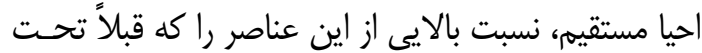

عمليات مكانيكى قرارگرفتهاند، حفظ خواهد كرد [24].

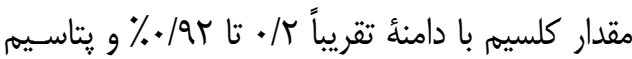

محصولات در شكل شماره ץ نشان دادهشه اسـت [18].

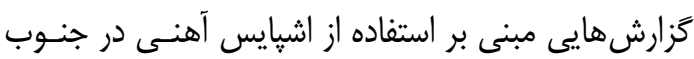
شرق ايران و در محوطــُ شـهر سـوخته، از هـزارهٔ ســوم،

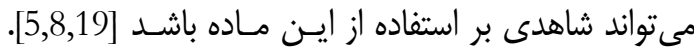
طرح اين موضوع كه در منطقهُ جنوب شرق فـلات ايـران

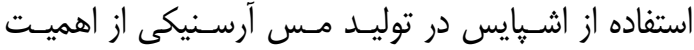

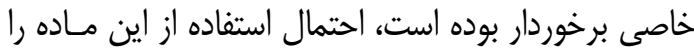
در منطقة اسييدز بزمان براى توليد مس آرسـنيكى، قـوّت

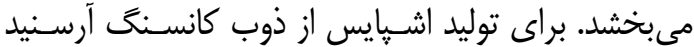

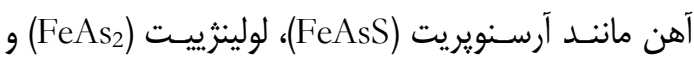


شناسايىشده است. اين عناصر بلعنــوان گَـداز آور

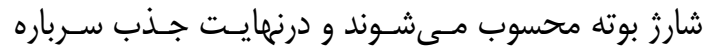

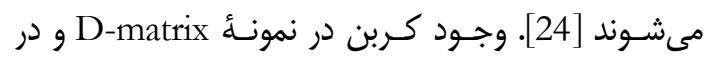

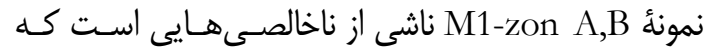

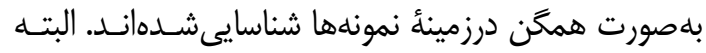

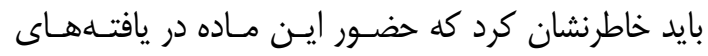
فلزى شهر سوخته نيز شناسايىشده است [5].

\section{"-1. بررسىهاى ريزساختارى}

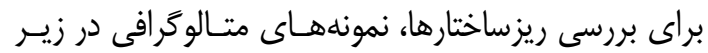
SEM-EDS

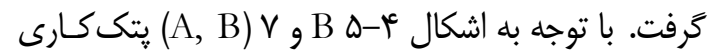

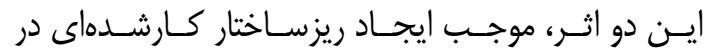
ناخالصىهاى آهن شده است كه اين ناخالصىها رفتهارفته

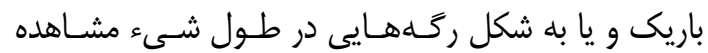
مىشوند. البته بايد موضوع مهمى را خاطرنشان كـرد كـهـ

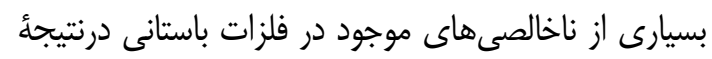

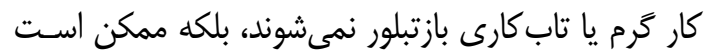

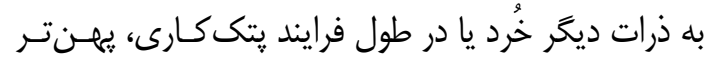
شوند كه اين فرايند در زمينأ دو تبر كاملاً مشهود است. در دو ساختار شكل ه-و حضور زياد ناخالصىهـا، بـاــا

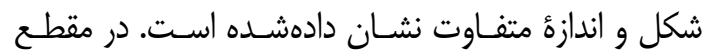
عرضى شكل م، B شكل مكعبى در قسمت بالاى شى

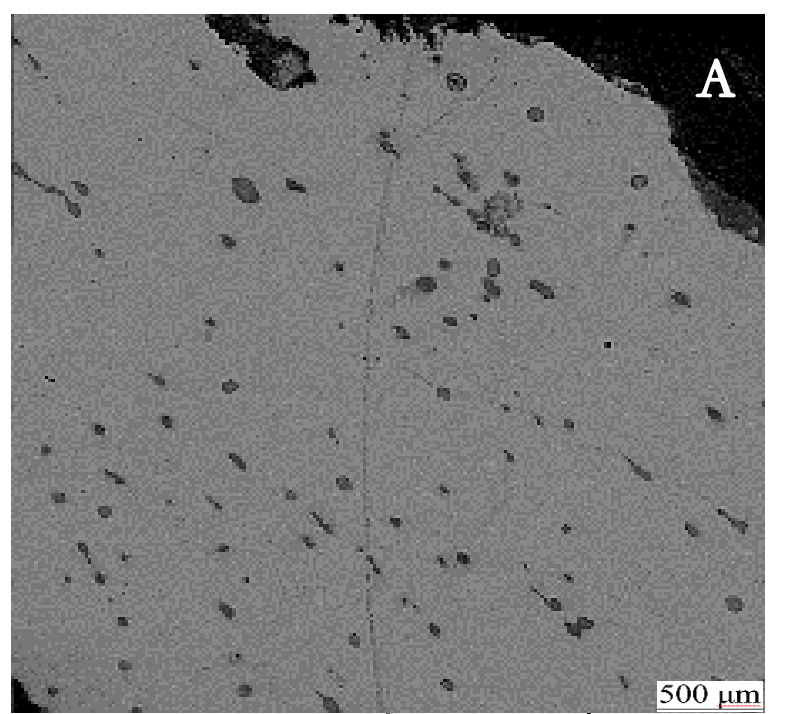

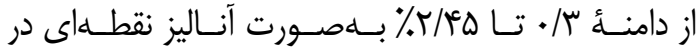

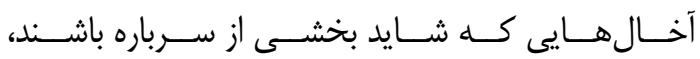

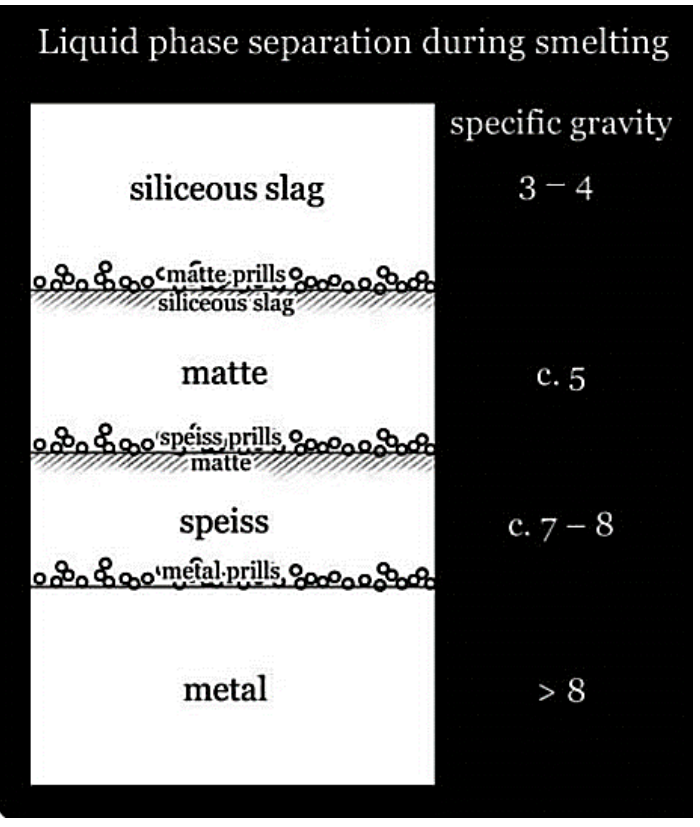

شكل س: طرح ساده Keesmann (1991) با توجه به وزن

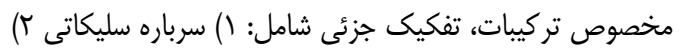

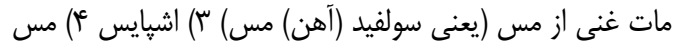
Fig. 3: Simple design by keesmann. According to the specific gravity of the compounds, the partial separation includes: 1-Silicate slag 2-Matte, i.e sulfide (Iron) of copper 3- Speiss 4- Copper metal

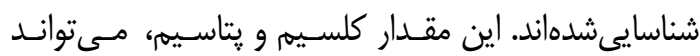

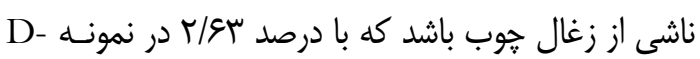

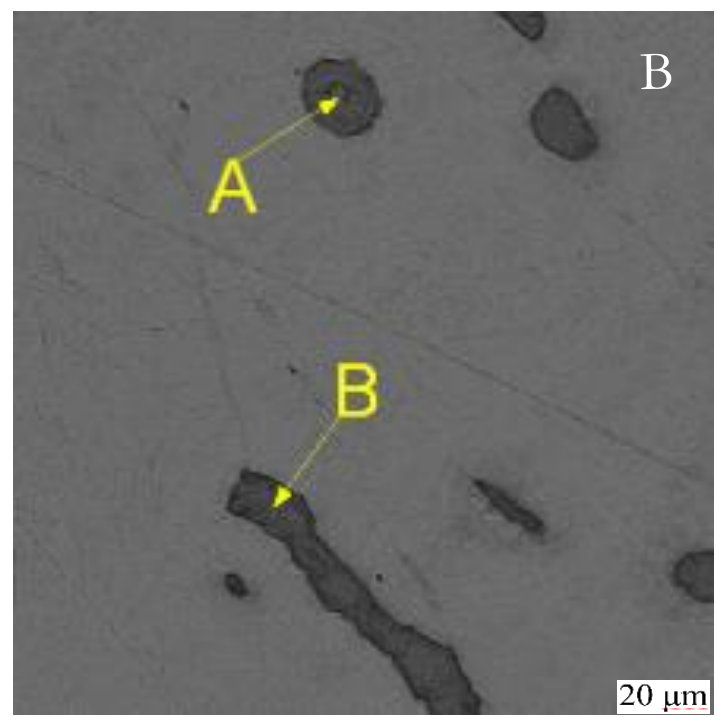

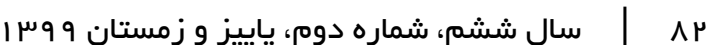




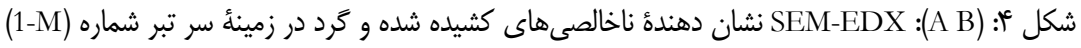

Fig. 4: (A, B). SEM-EDX represents stretched and round impurities in the field of axe head No.(1-M)
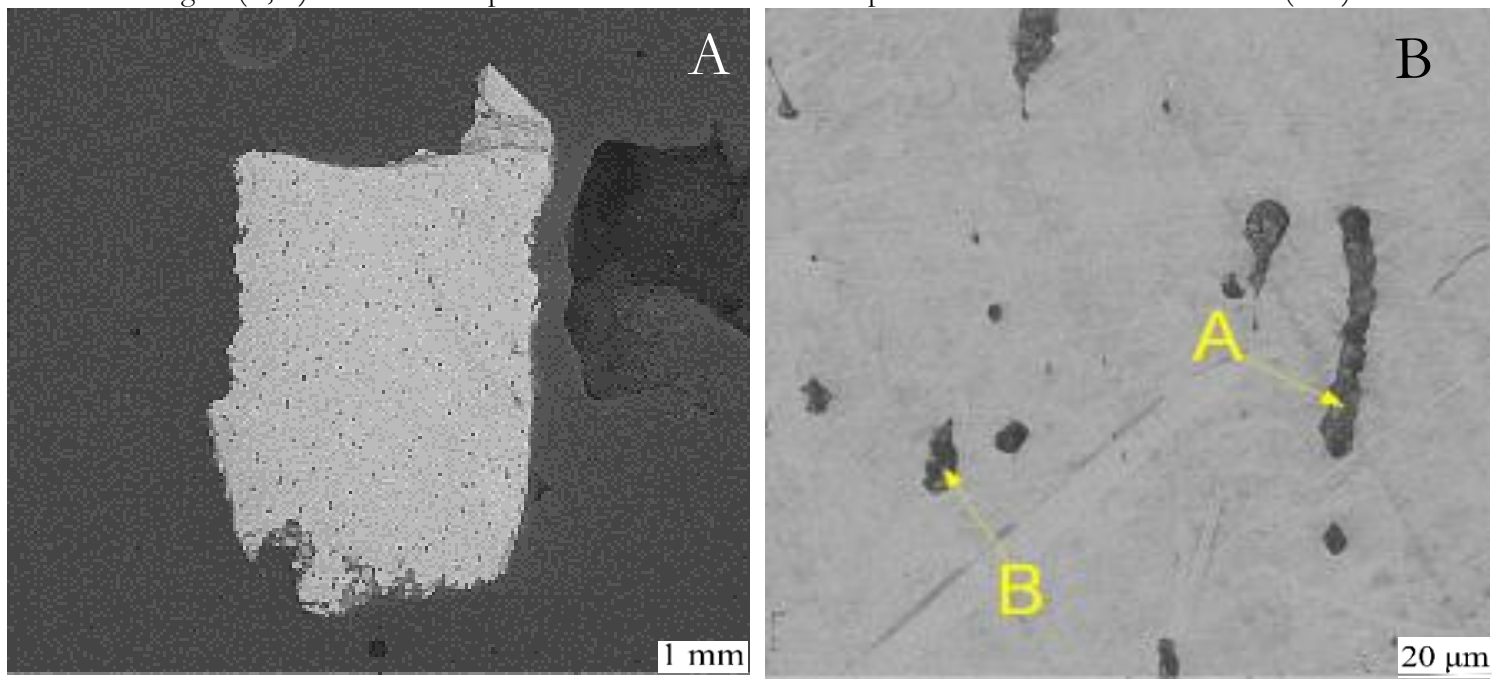

شكل ه: (A, B): نشان دهنده ناخالصىهاى كشيده شده و كرد در زمينه سر تبر شماره (A)-AEM-EDX)

5: (A, B). SEM-EDX Reperesents elongated and rounded impurities in the head No. (2-A) Matrix. Fig

ז ז در نمونهها شناسايى شد. وجود ناخالصى سولفور دليل بر استفاده از كانسنى مس سولفيدى براى استحال و توليد

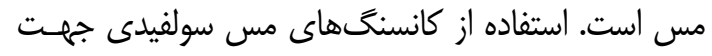

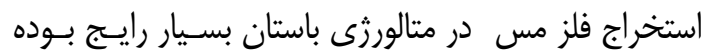

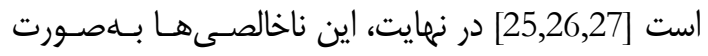

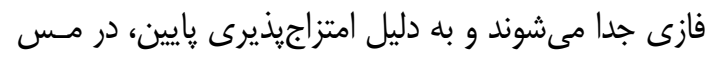
مذاب باقى مى مانند [28].
ديده مىشود كه با توجه به مقطع مكعبى آن بهاحتمالزياد،

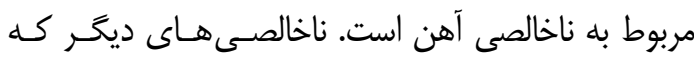

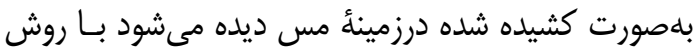
SEM- EDS نتايج، نشان مى دهند كه اين ناخالصـىهـا داراى تركيـب

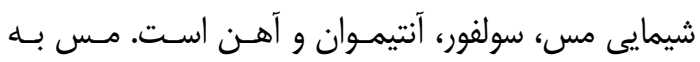

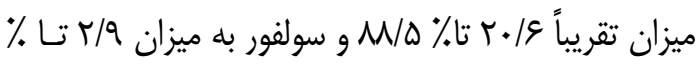
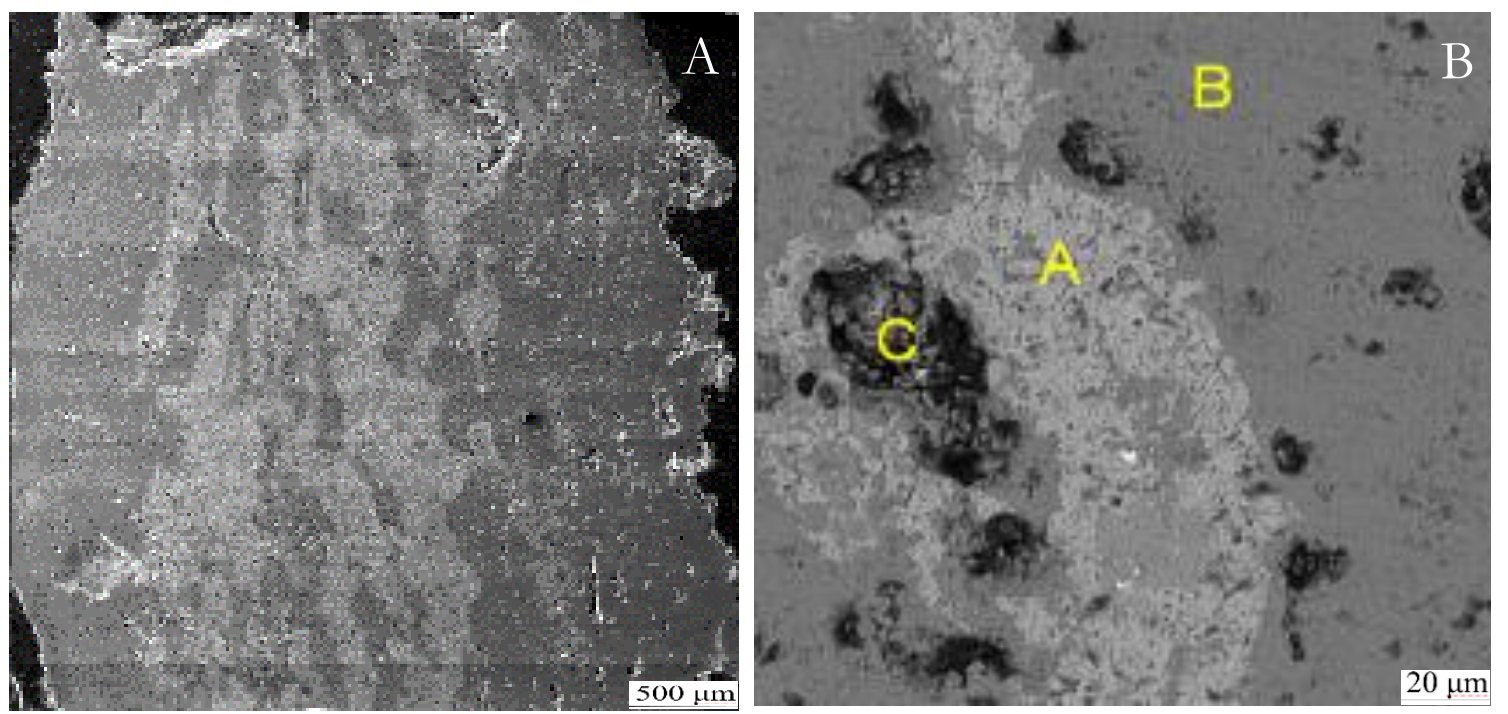

شكل S• نشان دهنده ناخالصىها و حفرات نامنظهم و وجود فاز خاكسترى روشن ناشى از خوردگى هاى كلريدى مس و

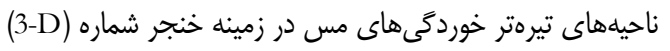


6: (A, B): SEM-EDX show incolosiobns and irregular cavities and the presence of a light gray phase caused by .Fig chlorinated corrosion of copper and darker areas of copper corrosion in the field of dagger No (3-D)
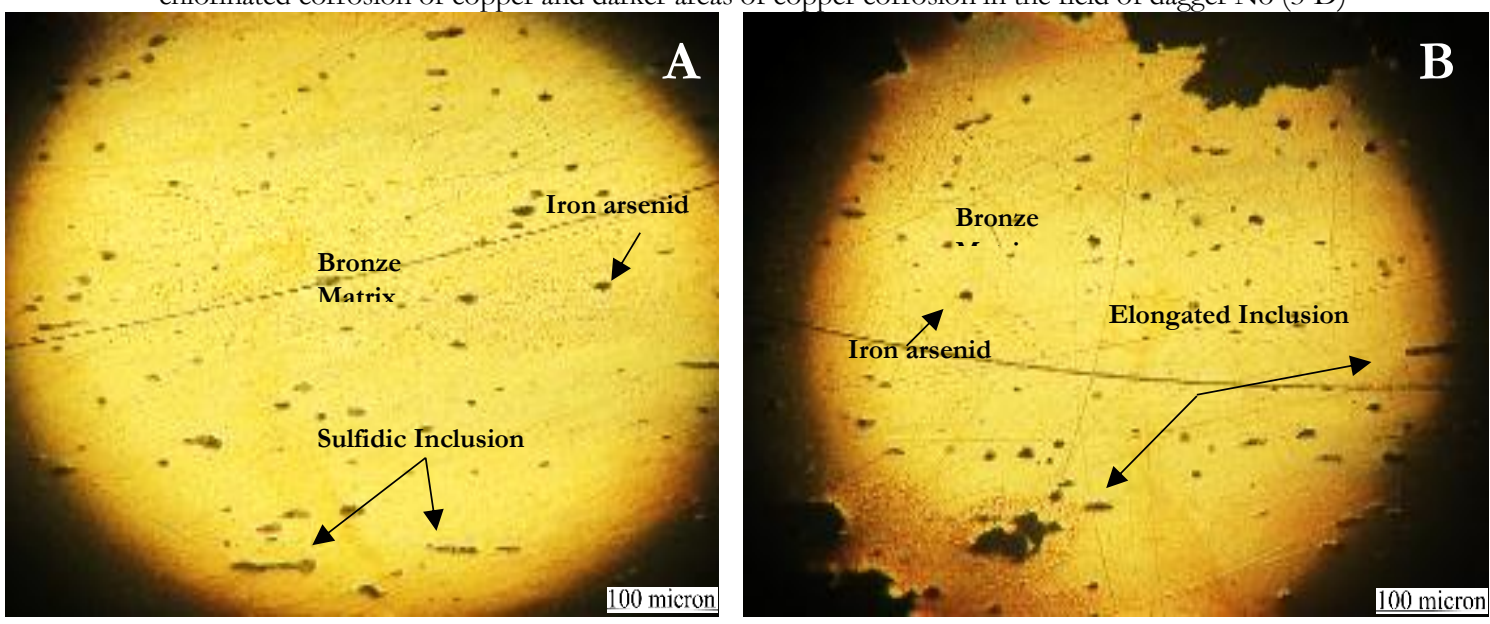

شكل V: ريز ساختار تبر (1-M) در شكل A و تبر (2-A) در شكل B قبل از حكاكى: شامل ناخالصىهاى كشيده سولفيد مس و آرسنيد آهن در زمينه برنز

Fig. 7: microstructure of axe (1-M) in figure A and Axe (2-A) in Figure Bbefore etching: including elongated impuritie of copper sulfide and iron arsenide in the bronze field

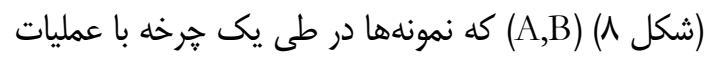

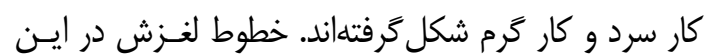

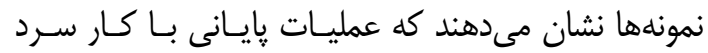

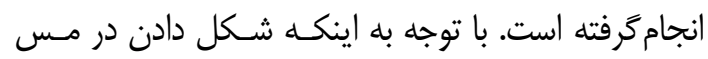

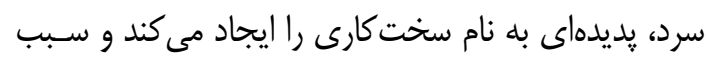
عدم فرم يذيرى بيشتر شـى مسىشـود، بنـابر اين، فلزكـار

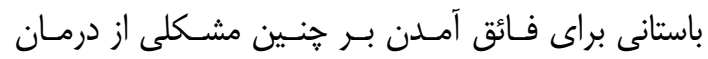

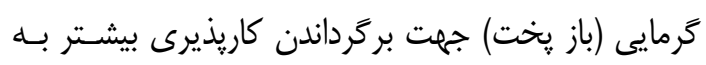

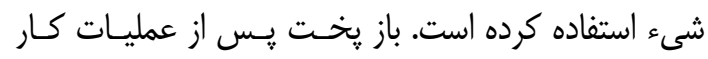

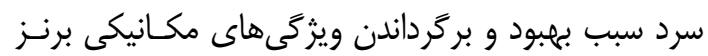

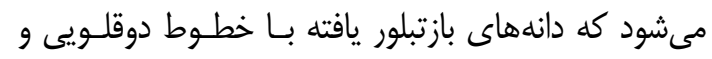

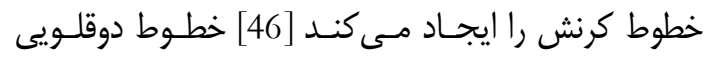

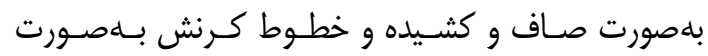

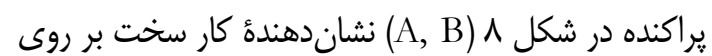
اين سر تبرها است. ساخت و توليد اين كونه تبرها، ازجمله دو تبـر موجـود

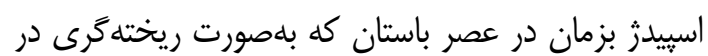

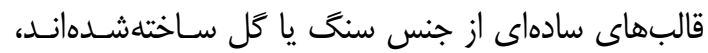

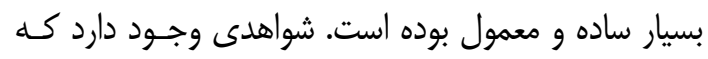

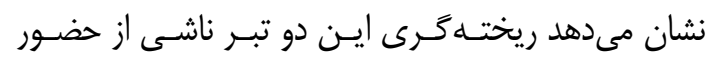

در نمونــهـهـــاى متــالوكر افى قبـــل از حكـــاكى،

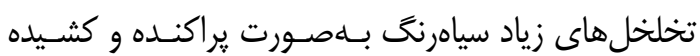

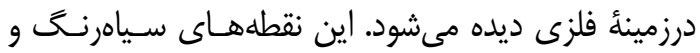
كشيده در شكل (A, B) مشخص ششدهاند. قسمت بالا

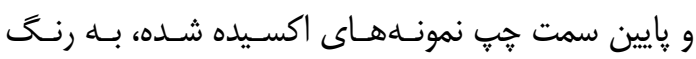

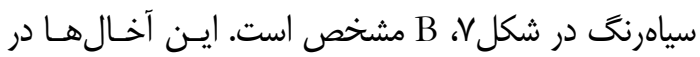

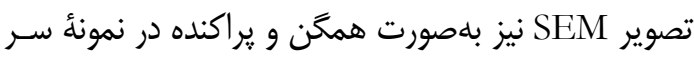

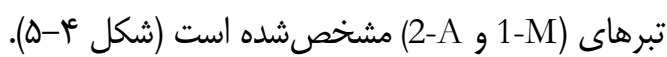
جهت بررسى و مطالعهُ ريزسـاختارها و سـاخت آليـاز موردنظر، نمونههاى آمادهشده با محلول اج

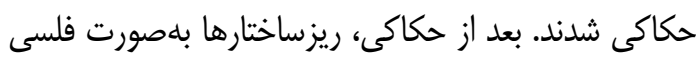

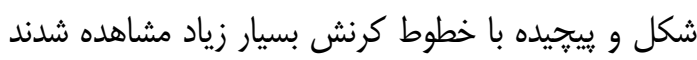

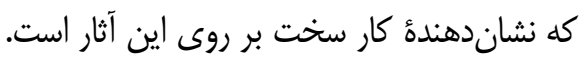

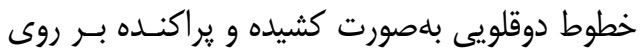

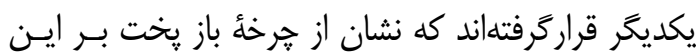

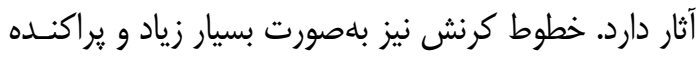

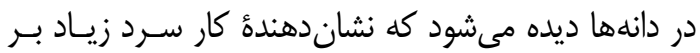
اين دو نمونه سر تبر است. اين ريزسـاختارها در آليازهـاى دئاي

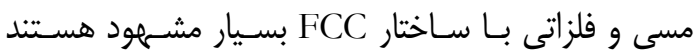

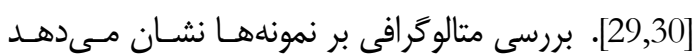




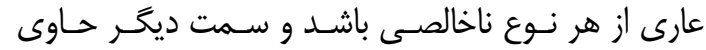

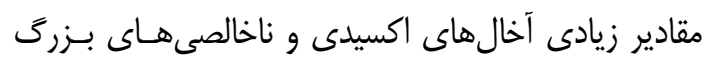

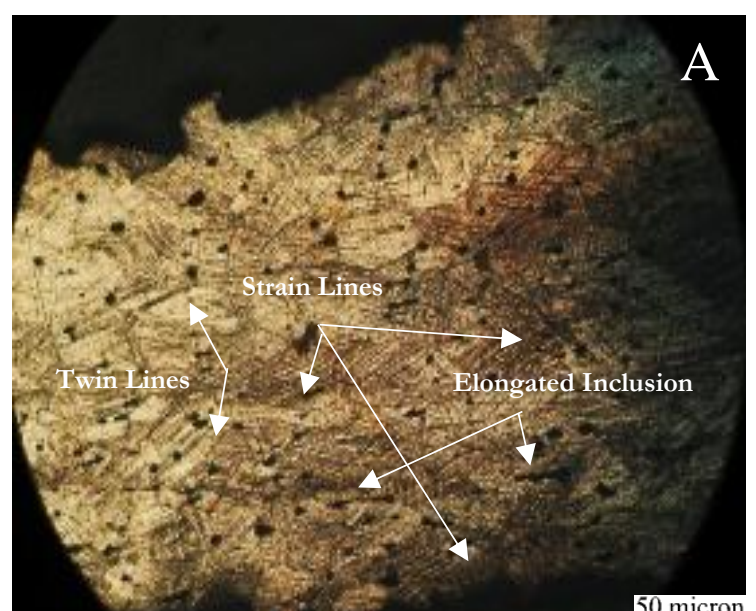

ناخالصىهايى است كه به سطحرويى قالب بـاز جـايش مىيابند كه ممكن است سبب شود يكطرف مقطع كاملاً

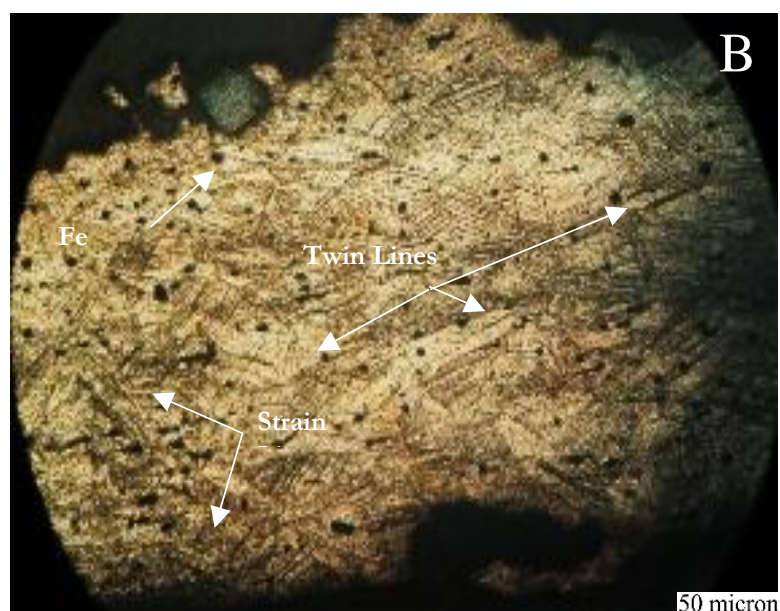

شكل م: ريز ساختارهاى تبر (1-M) و (A-2) بعد از حكاكى درمحلول FeCl الكلى. درتبر شمار (1-M) شكل A و سرتبر شماره (A-A) در شكل

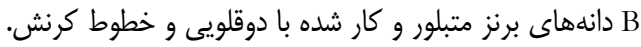

Fig. 8: Microstructures of axe (1-M) and (2-A) after etching in alcoholic solution $\mathrm{FeCl}_{3}$. In the image of axe No.(1-M) image A and in the image of axe No.(2-A) in image B. Crystallized and worked bronze grains with twins and strain lines

در آلياز مس آرسنيكى مانند آلياز برنز قلعى بـا ڤيديـده

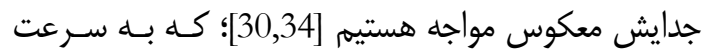

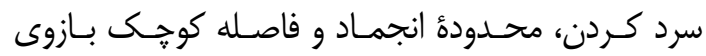

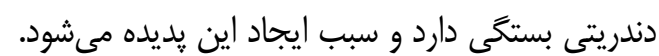

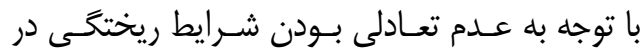

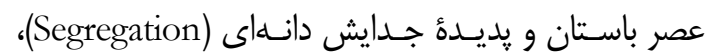
فشار ايجادشده توسط بخار آرسنيك سبب مسى وشـود گَاز

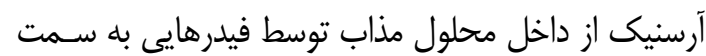

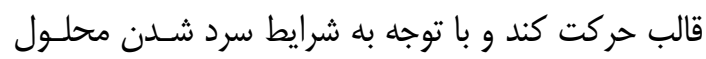

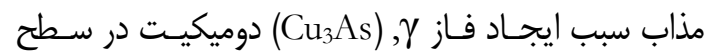

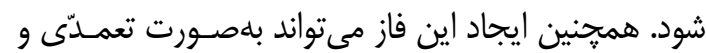

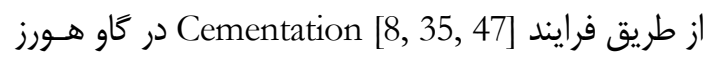

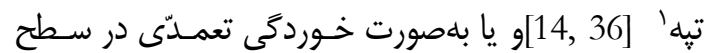
ايجاد شود [13, 37, 38].

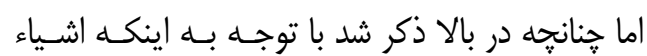
مس حاوى آرسنيك در عصر باستان بهصورت غير تعادلى بلى بله

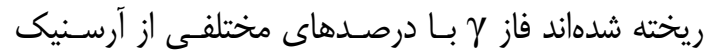

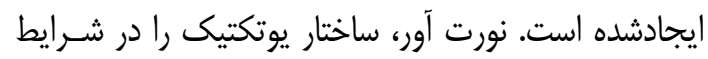

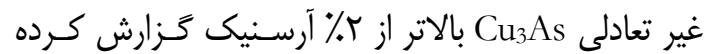
است [13, 34]. همجنين بود، يِيدايش نخسـتين فـاز ر در
ديگر به شكل سرباره ديده شود [30].

جنانج

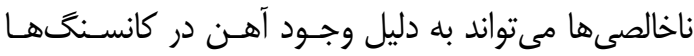

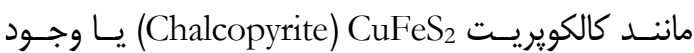
اشــايس در مـس باشــد. حضــور اكسـيد آهــن در ايـنـ ناخالصىها شاهدى بر وجود اين ادعا است.

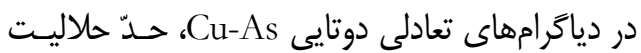

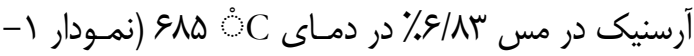

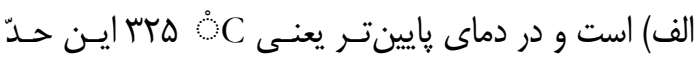

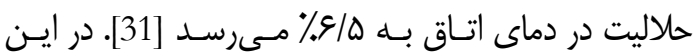

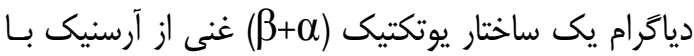

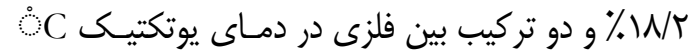

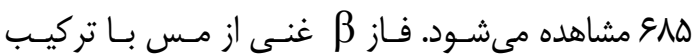
CusAs

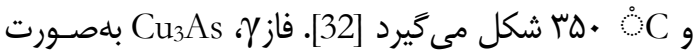

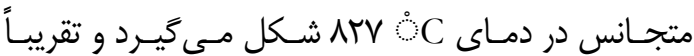

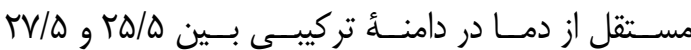

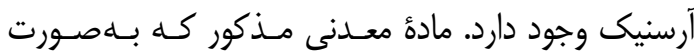

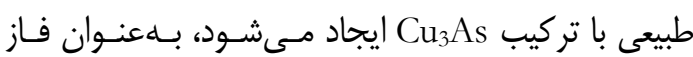
دوميكيت شناخته مى شود [33]. 


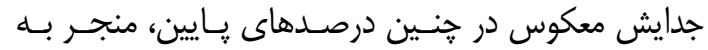

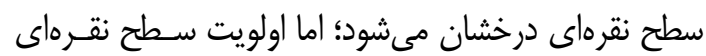

$w$ (As)
حدود ז\% تا 1\% آرسنيك را، مشاهده كرده است [39]. اين

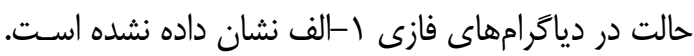
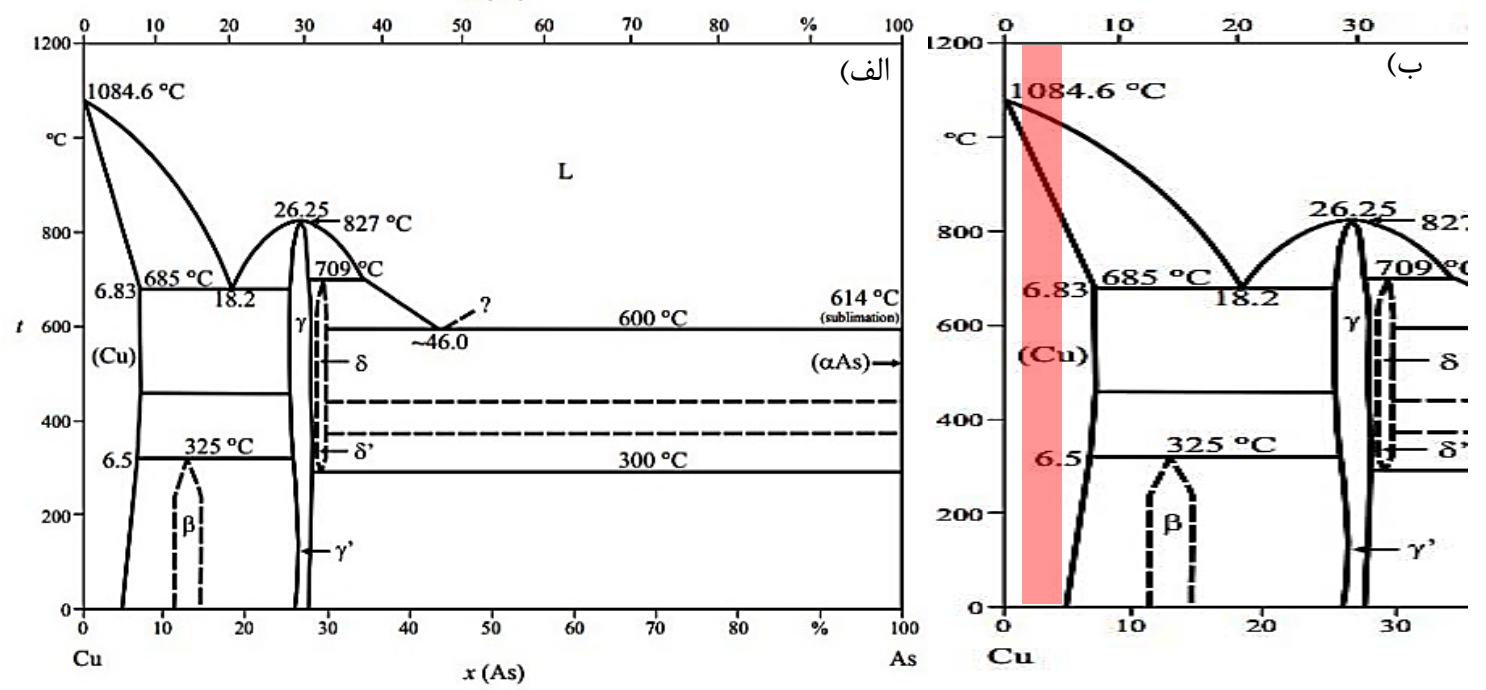

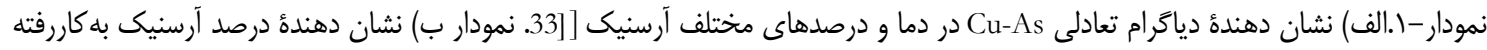

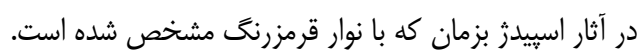

Fig. 1: A: shows the equilibrium diagram of $\mathrm{Cu}-\mathrm{As}$ at different temperatures and weight percentages of arsenic. Figure-1, B: indicates the weight percentage used in Spidej of Bazman artifacts, which is marked with a red bar

كه بر روى آثار اسيبيدز وجود دارد بهاحتمالزيـاد، سـبب از

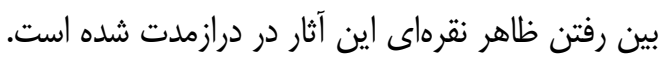
ع. نتيجه كَيرى با توجه به آناليز SEM-EDS بركيرى برى روى اشياء مشخص شد،

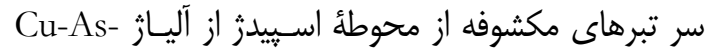

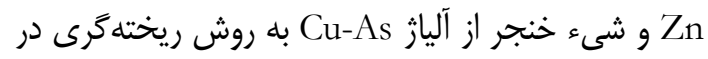

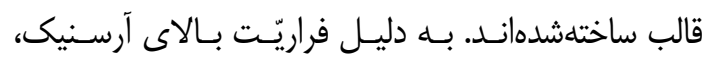

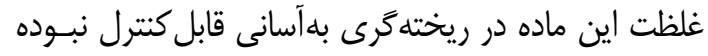

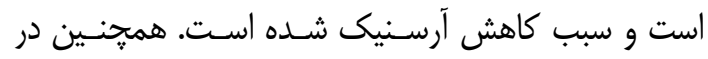

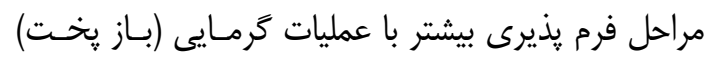

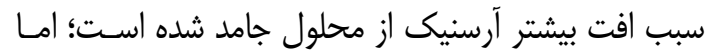
بههرحال درصد بالاى آرسنيك حـدود هـ در در ايـن اشـياء

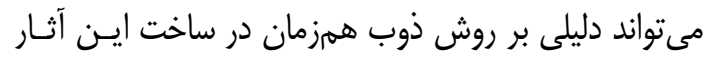

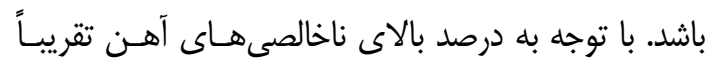

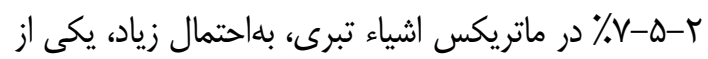

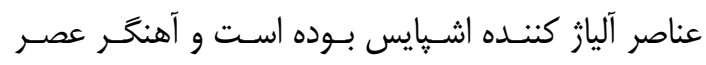

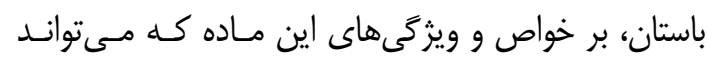
سبب يايين آوردن دماى نقطه ذوب آلياز شود، اطلاع لازم

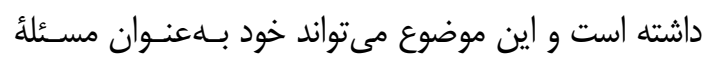

در اين آثار مىتواند دليلى بر ريختهَّى كنترلشده و

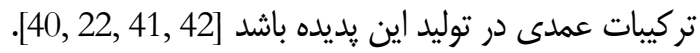

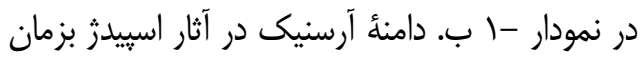
بر روى دياتر ام فـازى Cu-As بـا نـوار قرمزرنـــ نشـان دادهده است. مطابق دياتر ام فازى در شرايط ريختهَّى إنى

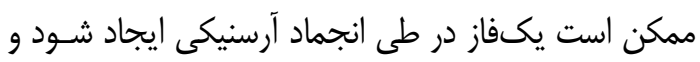

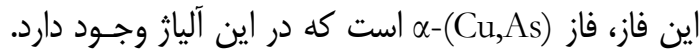

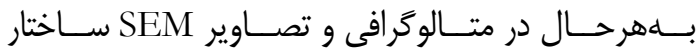

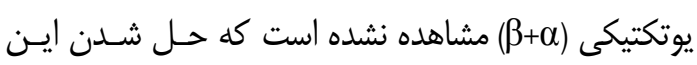

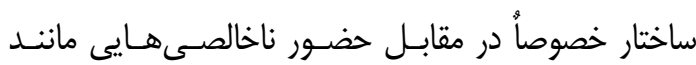

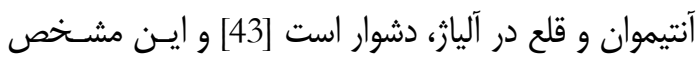
مى كند كه اين اشيا فاقد اين نوع ساختار هستند.

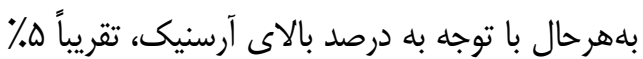

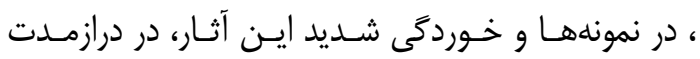

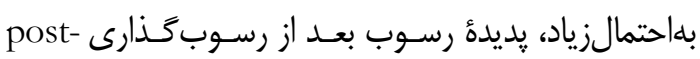
(depositional) precipitation

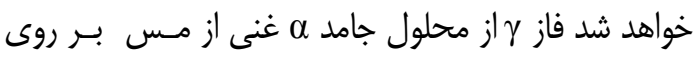

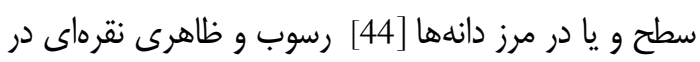

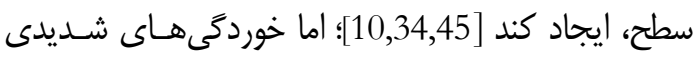

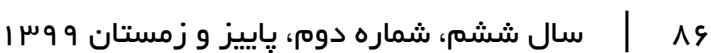




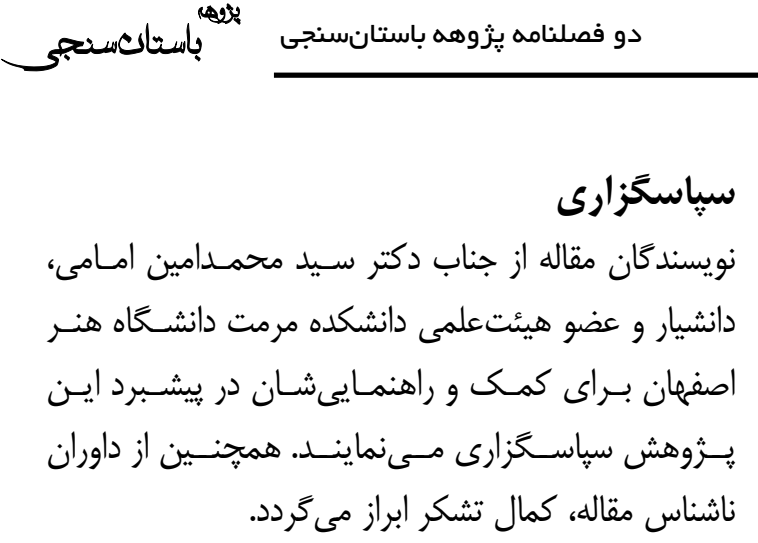

\section{References}

[1] Thornton CP, Lamberg-Karlovsky CC, Liezers M, Young SM. On pins and needles: tracing the evolution of copperbase alloying at Tepe Yahya, Iran, via ICPMS analysis of common-place items. Journal of Archaeological Science. 2002 Dec 1;29(12):1451-60.

[2] Lechtman H. Arsenic bronze: dirty copper or chosen alloy? A view from the Americas. Journal of field archaeology. 1996 Jan 1;23(4):477-514.

[3] Meier DM. Preliminary archaeometallurgical investigations of Bronze Age metal finds from Shahdad and Tepe Yahya. Iranian Journal of Archaeological Studies. 2011 Apr 1;1(2):25-34.

[4] Kashani P, Sodaei B, YOUSEFI R, Hamivand M. Arsenical Copper production in the Late-chalcolithic period, central plateau, Iran. Case study: Copperbased Artefacts in Meymanatabad. Interdisciplinaria Archaeologica, Natural Sciences in Archaeology. 2013:99-103.

[5] Hauptmann A, Rehren T, Schmitt-Strecker S. Early Bronze Age copper metallurgy at Shahr-i Sokhta (Iran) reconsidered. Deutsches Bergbau-Museum; 2003 Jan 29.

[6] Budd P, Gale D, Pollard AM, Thomas RG, Williams PA. The early development of metallurgy in the British Isles. Antiquity. 1992 Sep 1;66(252):677.

[7] Lechtman H. The manufacture of copperarsenic alloys in prehistory. Historical Metallurgy. 1985;19(1):141-2.

[8] Thornton CP, Rehren T, Pigott VC. The production of speiss (iron arsenide) during the Early Bronze Age in Iran. Journal of Archaeological Science. 2009 Feb 1;36(2):308-16.

[9] Rehren T, Schneider J, Bartels C. Medieval

$$
\begin{aligned}
& \text { مهمى جهت تأمين انرزى سوخت در اين محوطه باشد. از }
\end{aligned}
$$

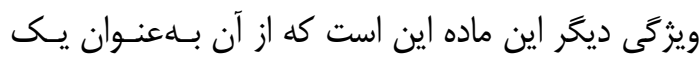

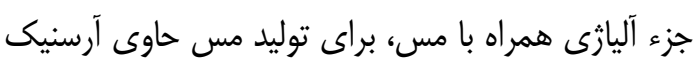

$$
\begin{aligned}
& \text { استفادهشده است. } \\
& \text { يعىنوشت } \\
& \text { ا. فرايندى كه در مجسمه كاو بهدستآمده از هورز تبه آناتولى إنى }
\end{aligned}
$$

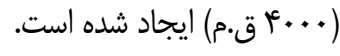

lead-silver smelting in the Siegerland, West Germany. na; 1999.

[10] Mödlinger M, Sabatini B. A Re-evaluation of inverse segregation in prehistoric As$\mathrm{Cu}$ objects. Journal of Archaeological Science. 2016 Oct 1;74:60-74.

[11] Momeni, N. Emami, S. M. A., Karimy, A. M. Technology and comparsion of two types of gray and red wares from Espidezh cemetery of Bazman in Baluchistan according to the samples deterioration, Iahan. M.A. thesis. Art university of Isfahan, 2018.pp 9-10

[12] Pourzarghan, V., Sarhadi-Dadian, H. Hosseini, S. Identifying the Technology of a Bronze Dagger Discovered in Espidezh Region of Bazman in Sistan and Baluchestan, Iran. Journal of Anthropology and Archaeology June 2017; 5(1):47-54.

[13] Northover JP. Properties and use of arsenic-copper alloys. Old world archaeometallurgy. 1989:111-8.

[14] Scott DA. Copper and bronze in art: corrosion, colorants, conservation. Getty publications; 2002.

[15] Craddock PT, Meeks ND. Iron in ancient copper. Archaeometry. 1987 Aug; 29(2):187-204.

[16] Craddock, P. The Composition of the copper alloys used by the Greek, Etruscan and Roman civilisations: The Greeks before the Archaic Period. Journal of Archaeological Sciences. (1976) 3, pp93113.

[17] Cowell MR. Scientific appendix I: chemical analysis. InCatalogue of Egyptian antiquities in the British Museum, VII, tools and weapons, I, axes 1987 (pp. 96118). 
[18] Keesmann I, Onorato AM. Naturwissenschaftliche Untersuchungen zur frühen Technologie von Kupfern und Kupfer-Arsen-Bronze. na; 1999.

[19] Doonan RC, Day PM, DimopoulouRethemiotaki N. Lame excuses for emerging complexity in Early Bronze Age Crete: the metallurgical finds from Poros Katsambas and their context. Metallurgy in the early bronze age aegean. 2007:98122.

[20] Merkel JF, Shimada I, Swann CP, Doonan R. Pre-Hispanic copper alloy production at Batan Grande, Peru: interpretation of the analytical data for ore samples. InArchaeometry of pre-Columbian sites and artifacts: proceedings of a symposium organized by the UCLA Institute of Archaeology and the Getty Conservation Institute, Los Angeles, California, March 23-27, 19921994 Oct 27 (pp. 199-227).

[21] Petersen, Ulrich. Geological Framework of Andean Mineral Resources. In George E. Ericksen, Maria Theresa Cafias P., and John A. Reinemund, eds., Geology of the Andes and Its Relation to Hydrocarbon and Mineral Resources. CircumPacific Council for Energy and Mineral Resources, Earth Science Series 11. Houston: Circum-Pacific Council for Energy and Mineral Resources, 1989: 213232.

[22] Rostocker W, Dvorak JR. Some experiments with co-smelting to copper alloys. Archeomaterials. 1991;5(1):5-20.

[23] Nazari AM, Radzinski R, Ghahreman A. Review of arsenic metallurgy: Treatment of arsenical minerals and the immobilization of arsenic. Hydrometallurgy. 2017 Dec 1;174:258-81.

[24] Tylecote RF, Ghaznavi HA, Boydell PJ. Partitioning of trace elements between the ores, fluxes, slags and metal during the smelting of copper. Journal of Archaeological Science. 1977 Dec 1;4(4):305-33.

[25] Coghlan HH. Notes on the prehistoric metallurgy of copper and bronze in the Old World.

[26] Bachmann HG. The identification of slags from archaeological sites. Routledge; 2016 Jun 16.

[27]Hauptmann A. The investigation of archaeometallurgical slag.
InArchaeometallurgy in Global Perspective 2014 (pp. 91-105). Springer, New York, NY.

[28] Valério P, Soares AM, Araújo MF, Silva RJ, Porfírio E, Serra M. Arsenical copper and bronze in Middle Bronze Age burial sites of southern Portugal: the first bronzes in Southwestern Iberia. Journal of Archaeological Science. 2014 Feb 1;42:6880.

[29] Figueiredo E, Araújo MF, Silva RJ, Sennamartinez J. Corrosion of bronze alloy with some lead content: implications in the archaeometallurgical study of Late Bronze Age metal artefacts from "Fraga dos Corvos"(North Portugal). METAL07 (ICOM-CC) Proceedings, Amsterdam. 2007;1:61-6.

[30] Scott DA. Metallography and microstructure in ancient and historic metals. Getty publications; 1992 Jan 2.

[31] Dies K. Herstellungsverfahren von Kupfer und Kupferlegierungen (Ausgewählte Abschnitte). InKupfer und Kupferlegierungen in der Technik 1967 (pp. 67-100). Springer, Berlin, Heidelberg.

[32] Subramanian, P.R, Laughlin, D.E, As-Cu (Arsenic copper). In Subramanian, P.R,.editor, phase diagrams. 1994: 43-52. The materials information Society, Materials Park. Ohio.

[33] Junk M. Material properties of copper alloys containing arsenic, antimony, and bismuth.

[34] Meeks N. Surface characterization of tinned bronze, high-tin bronze, tinned iron and arsenical bronze. InMetal plating and patination 1993 Jan 1 (pp. 247-275). Butterworth-Heinemann.

[35] Eaton ER, McKerrell H. Near Eastern alloying and some textual evidence for the early use of arsenical copper. World Archaeology. 1976 Oct 1;8(2):169-91.

[36] Ogilvie RE. A Few Examples of Electron Microanalysis of Art Objects at the Boston Museum of Fine Arts. Microscopy and Microanalysis. 2001 Mar;7(2):193-199.

[37] Budd P. A metallographic investigation of Eneolithic arsenical copper artefacts from Mondsee, Austria. Historical metallurgy. 1991;25(2):99-108.

[38] Budd, P.D, determination of the manufacturing processes of early prehistoric arsenical copper artefacts by

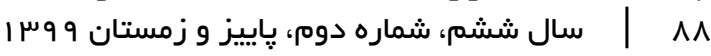


microstructural analysis based on Reevaluation of mechanical properties of copper -Arsenic alloys (unpublished phd, university of Bradford). 1991b.

[39] Budd P. Eneolithic arsenical copper: heat treatment and the metallographic interpretation of manufacturing processes. Archaeometry. 1991;90:35-44.

[40] Lechtman H. The manufacture of copperarsenic alloys in prehistory. Historical Metallurgy. 1985;19(1):141-2.

[41] Pollard AM, Thomas RG, Williams PA. Some experiments concerning the smelting of arsenical copper. Archaeological Sciences. 1989 Sep:169-74.

[42] Giumlia-Mair A, Keall EJ, Shugar AN, Stock S. Investigation of a copper-based hoard from the Megalithic site of alMidamman, Yemen: an interdisciplinary approach. Journal of Archaeological Science. 2002 Feb 1;29(2):195-209.

[43] La Niece S, Carradice I. White copper: the arsenical coinage of the Libyan revolt 241238 BC. Historical metallurgy. 1989;23(1):9-15.

[44] Pereira F, Silva RJ, Soares AM, Araújo MF,
Oliveira MJ, Martins RM, Schell N. Effects of long-term aging in arsenical copper alloys. Microscopy and Microanalysis. 2015 Dec 1;21(6):1413.

[45] Emami, S.M.A, Jafari, J. Archaemetallugical studies on corrosion behavior of Cu-As alloys from LAMA (2nd millennium BC), Iran In: Arzarello, Marta. Djindjian, François. Oosterbeek, Luiz. (Eds) - UISPP Journal The Journal of the International Union of Prehistoric and Protohistoric Sciences. 2019. Vol 2-1: pp24-32.

[46] Figueiredo E, Senna-Martinez JC, Silva RJ, Araújo MF. Orientalizing artifacts from Fraga dos Corvos rock shelter in North Portugal. Materials and Manufacturing Processes. 2009 Jul 24;24(9):949-54.

[47] Smith CS. An examination of the arsenicrich coating on a bronze bull from Horoztepe. InApplication of science in examination of works of art-proceedings of the seminar: June 15-19, 1970, conducted by the research laboratory, Museum of Fine Arts, Boston, Massachussetts 1973 (pp. 96-102). 